\title{
Imperfections of the Three-Dimensional Thermohaline Circulation: Hysteresis and Unique-State Regimes*
}

\author{
Henk A. Dijkstra And Wilbert Weijer \\ Institute for Marine and Atmospheric Research Utrecht, Department of Physics and Astronomy, Utrecht University, Utrecht, Netherlands \\ J. DAVID NeElin \\ Department of Atmospheric Sciences, and Institute for Geophysics and Planetary Physics, University of California, Los Angeles, \\ Los Angeles, California
}

(Manuscript received 9 July 2002, in final form 2 May 2003)

\begin{abstract}
Different equilibria of oceanic thermohaline circulation may exist under the same forcing conditions. One of the reasons for the existence of these multiple equilibria is a feedback between the overturning circulation and the advective transport of salt and heat. In an equatorially symmetric configuration, the multiple equilibria arise through symmetry-breaking pitchfork bifurcations when the strength of the freshwater forcing is increased. Here, continuation methods are used to track the fate of the different equilibria under equatorially asymmetric conditions in a three-dimensional, low-resolution ocean general circulation model in an Atlantic-like basin coupled to an energy-balance atmosphere model. The effect of the continental geometry, the presence of the Antarctic Circumpolar Current (ACC), and asymmetric air-sea interaction on the preference of equilibria are considered. Although all asymmetry-inducing mechanisms favor northern Atlantic sinking states, the open Southern Ocean and ACC are shown to be substantial contributors. The origin of the hysteresis behavior between strong and weak overturning states is clarified in terms of the overall bifurcation picture. The disappearance of a class of southern sinking equilibria because of the combined effects of all asymmetry mechanisms leads to a substantial regime with a unique steady state. The relationship between the hysteresis regime and the unique-state regime provides a larger context for quantitative determination of the relevance of each to climate.
\end{abstract}

\section{Introduction}

Reconstruction of temperature records from ice cores and ocean sediments indicates that relatively rapid climate transitions have occurred in the past. One of the long-standing hypotheses of these rapid transitions is changes in the ocean circulation, in particular in that part of the circulation which is induced by the surface buoyancy forcing, that is, the thermohaline circulation (THC) (Broecker 1995). In a hierarchy of models, from simple box models (Stommel 1961) up to global coupled GCMs (Manabe and Stouffer 1988), existence of multiple thermohaline flow patterns under the same forcing conditions are found. Hence, it is tempting to relate the rapid climate changes in the past to transitions between

\footnotetext{
* Institute for Geophysics and Planetary Physics Contribution Number 5762 .
}

Corresponding author address: Henk A. Dijkstra, Institute for Marine and Atmospheric Research Utrecht, Department of Physics and Astronomy, Utrecht University, Princetonplein 5, 3584 CC Utrecht, Netherlands.

E-mail: dijkstra@phys.uu.nl different equilibria of the thermohaline circulation (Rahmstorf 2000).

When an equatorially symmetric Atlantic basin is subject to mixed boundary conditions, it is known that symmetry-breaking pitchfork bifurcations are at the origin of multiple equilibria (Bryan 1986; Thual and McWilliams 1992; Cessi and Young 1992; Quon and Ghil 1992; Dijkstra and Molemaker 1997; Weijer and Dijkstra 2001). The physics of this pitchfork bifurcation is also well known: the symmetric thermally driven state becomes unstable to asymmetric salinity perturbations when the freshwater forcing is large enough. These perturbations modify the overturning circulation in such a way that perturbations in the advection of salt amplify the original salinity perturbation (Marotzke et al. 1988; Quon and Ghil 1992; Weijer et al. 2001). This symmetry breaking leads to asymmetric Pole-to-Pole solutions that can be maintained under equatorially symmetric forcing conditions. In ocean GCMs with idealized geometry, this type of symmetry breaking is responsible for the existence of northern and southern sinking solutions (Bryan 1986; Marotzke and Willebrand 1991; Klinger and Marotzke 1999).

In ocean GCMs, transitions are also found between 
a strong and a weak (or absent) Atlantic overturning circulation as the freshwater strength or pattern is changed, and hysteresis effects have been found in these transitions (Rahmstorf 1995; Stocker 2000). The transient behavior associated with the hysteresis has become a paradigm to explain transitions in the (glacial) past (Broecker 1995; Stocker 2000; Ganopolsky et al. 2001) and possible transitions of the future circulation (Tziperman 2000; McAvaney 2001). At first sight, there is no apparent relation with these transitions and the multiple equilibria in the equatorially symmetric situation (Marotzke 2000).

The key to relate these equilibria is to consider systematically the effects of asymmetry on the multipleequilibria structure that arises under equatorially symmetric forcing. A useful way of investigating this is to consider bifurcation diagrams in which properties of solutions are plotted versus one of the parameters of the system. Each of the asymmetries will break the pitchfork bifurcations that are present in the equatorially symmetric situation and, as a result, disconnected branches of steady solutions occur. For an easy introduction into the most elementary bifurcations, such as the pitchfork and the saddle-node bifurcation, the reader is referred to Seydel (1994), Nayfeh and Balachandran (1995), and Dijkstra (2000).

The effects of the asymmetries on the equilibria structure due to the surface freshwater flux, the continental geometry, and distribution of internal sources of heat and salt have been considered within two-dimensional models. From these studies, two main results were obtained.

- Strong preferences for some of the equilibria may occur. In this case, one of the disconnected branches of solutions moves out of the "realistic" range of parameter space or disappears through an isola. For example, the transport of salt and heat in the South Atlantic was shown to introduce a strong preference for the northern sinking solution (Weijer et al. 1999, 2001).

- New equilibria may occur, through the appearance of saddle-node bifurcations on either of the branches. For example, asymmetric air-sea interaction and an asymmetric freshwater flux also promote the northern sinking solution, but in a regime of weak freshwater forcing, two of these solutions (with weak and strong overturning) appear (Dijkstra and Neelin 2000).

One of the severe limitations in the Weijer et al. (2001) and Dijkstra and Neelin (2000) studies at first sight appears to be the two-dimensionality of the thermohaline flow. The momentum balances of the overturning flow in this case are between frictional processes and pressure gradients, whereas in the three-dimensional flow these are predominantly geostrophic. Precise identification of the physics of the different equilibria through the hierarchy of models, from two-dimensional Boussinesq via zonally averaged up to three-dimen- sional models, has been accomplished only for the equatorially symmetric case. Fortunately, bifurcation diagrams in the three-dimensional case are qualitatively the same as in the two-dimensional case. The instability of the thermally driven flow still occurs and does not depend on the details of the momentum balances of the buoyancy driven flow (Weijer and Dijkstra 2001).

However, in two-dimensional models, the additional asymmetry-inducing effects of the wind stress and the presence of the Antarctic Circumpolar Current (ACC) cannot be adequately captured. Here, we undertake a study to capture these effects in a three-dimensional model and simultaneously try to determine which factors are important for a preference of the asymmetric (northern sinking) overturning flow in the Atlantic ocean. The bifurcation diagrams are computed within a low-resolution ocean GCM coupled to an energy-balance atmosphere model. The model includes a rough representation of the Atlantic continental geometry and a representation of the ACC flow. Continuation methods are used to solve directly for the path of the steady-state solutions in parameter space. This is the first time that direct bifurcation studies are applied to such a detailed coupled three-dimensional ocean-atmosphere model (described in section 2).

The results follow a step-by-step approach from simple configurations to a more realistic situation with the various factors producing asymmetry about the equator added sequentially. In section 3, cases are examined in an idealized geometry, beginning with an equatorially symmetric sector basin similar to the Atlantic in size. Asymmetry about the equator is first introduced by opening the Southern Ocean boundaries. Next, wind stress forcing is added (based on the observed zonal average), the effects of which are most important in the ACC (Toggweiler and Samuels 1995; Rahmstorf and England 1997). Physical mechanisms for these impacts are discussed in section 3 before turning to a more complex, realistic Atlantic configuration in section 4, where all asymmetry mechanisms, including freshwater flux and bottom topography, are included (section 5). The combination of these results with inferences from the idealized geometry cases are used in the discussion (section 6) to sketch a global relation between the bifurcation diagram known from simpler models in the symmetric case and the segments of bifurcation diagrams pieced together from time integrations of more complex models. The main focus is on the origin of the two regimes, a hysteresis and a unique-state regime, that survive under the realistic equatorial asymmetry. A second, related focus is the impact of the open Southern Ocean and ACC on the bifurcation behavior of the THC.

\section{The coupled ocean-atmosphere model}

The ocean model is an extension of the fully implicit ocean model as presented in Dijkstra et al. (2001). In this version, continental geometry as well as bottom 
topography are implemented and the upper ocean is coupled to a simple energy-balance atmosphere model. Although the mixing representations in the model are still substantially less sophisticated than those in present low-resolution GCMs, the coupled model is by far the most complex system used for direct bifurcation studies of the THC to date. In the applications here, the model is used in a manner complementary to time-marched model studies (Rahmstorf and England 1997; Tziperman 2000) to provide an overall view of the relation among different stationary states of the THC. The results may be anticipated to be directly relevant to studies in low-resolution ocean-atmosphere GCMs, at least for questions that do not have highly sensitive dependence on mixing parameterizations.

\section{a. The ocean model}

Consider a sector flow domain $\left[\phi_{W}, \phi_{E}\right] \times\left[\theta_{S}, \theta_{N}\right]$ representing an ocean basin on a sphere with radius $r_{0}$. The basin has maximum depth $D$ and is rotating with angular frequency $\Omega$. The ocean velocities in eastward and northward directions are indicated by $u$ and $v$, the vertical velocity is indicated by $w$, the pressure by $p$, and the temperature and salinity by $T$ and $S$, respectively. Vertical and horizontal mixing of momentum and of heat and salt is represented by (constant) eddy diffusivities with horizontal and vertical friction coefficients $A_{H}$ and $A_{V}$ for momentum and horizontal and vertical diffusivities $K_{H}$ and $K_{V}$ for heat, respectively. The mixing coefficients of salt are taken equal to those of heat. A linear equation of state is taken with expansion coefficients $\alpha_{T}$ and $\alpha_{S}$ and reference temperature $T_{0}$, salinity $S_{0}$, and density $\rho_{0}$. To explicitly show which equations are actually solved, the governing equations are presented in appendix A.

The ocean circulation is driven by a wind stress $\boldsymbol{\tau}(\phi, \theta)=\tau_{0}\left(\tau^{\phi}, \tau^{\theta}\right)$, where $\tau_{0}$ is the amplitude and $\tau^{\phi}(\phi, \theta)$ and $\tau^{\theta}(\phi, \theta)$ provide the spatial pattern. The thermohaline component of the circulation is driven by heat and freshwater fluxes at the surface, with downward heat flux $Q_{o a}$. The freshwater flux is converted into an equivalent salt flux and is simply a prescribed (dimensionless) function $F_{S}(\phi, \theta)$ with (dimensional) amplitude $F_{0}$. As in low-resolution GCMs, the surface forcing is represented as a body forcing over the first layer of thickness $H_{m}$. Using these source terms, the appropriate boundary conditions for temperature, salinity, and wind stress at the ocean-atmosphere boundary are no-flux conditions. On the continental boundaries, no-slip conditions are prescribed and the heat and salt fluxes are zero. At the bottom of the ocean, both the heat and salt flux vanish and slip conditions are assumed.

\section{b. The atmosphere model}

In the energy-balance atmosphere model (North et al. 1981; Fanning and Weaver 1996), the equation for the atmospheric surface temperature $T_{a}$ on the domain $\left[\phi_{W}, \phi_{E}\right] \times\left[\theta_{S}, \theta_{N}\right]$ is given by

$$
\begin{aligned}
\rho_{a} H_{a} & C_{p a} \frac{\partial T_{a}}{\partial t} \\
= & \rho_{a} H_{a} C_{p a} D_{0} \nabla\left[D(\theta) \nabla T_{a}\right] \\
& -\left(A+B T_{a}\right)+\frac{\Sigma_{0}}{4} S(\theta)(1-\alpha)\left(1-C_{0}\right) \\
& +\mu_{o a}(1-\mathcal{L})\left(T-T_{a}\right)+\mu_{l a} \mathcal{L}\left(T_{l}-T_{a}\right),
\end{aligned}
$$

where $\rho_{a}$ is the atmospheric density, $C_{p a}$ is the heat capacity, $\alpha$ is the albedo, $H_{a}$ is an atmospheric scale height, $\Sigma_{0}$ is the solar constant, $D_{0}$ is a constant eddy diffusivity, and $1-C_{0}$ is the atmospheric absorption coefficient. The functions $D(\theta)$ and $S(\theta)$ give the latitude dependence of the eddy diffusivity and the shortwave radiative heat flux. The constants $A$ and $B$ control the magnitude of the longwave radiative flux. Last, $T$ is the sea surface temperature, $T_{l}$ is the temperature of the land surface points, and the coefficient $\mathcal{L}$ indicates whether the underlying surface is ocean $(\mathcal{L}=0)$ or land $(\mathcal{L}=1)$.

The exchange of heat between atmosphere and ocean and between atmosphere and the land surface is modeled by constant exchange coefficients. We assume here for simplicity that both are equal, with

$$
\mu_{l a}=\mu_{o a}=\rho_{a} C_{p a} C_{H}\left|V_{a}\right| \equiv \mu,
$$

where $C_{H}=1.22 \times 10^{-3}$ and $\left|V_{a}\right|$ is a mean atmospheric surface wind speed. With $\left|V_{a}\right|=2.5 \mathrm{~m} \mathrm{~s}^{-1}$, it follows that $\mu \approx 4 \mathrm{~W} \mathrm{~m}^{-2} \mathrm{~K}^{-1}$.

Boundary conditions for the atmosphere are periodic in zonal direction and no-flux conditions at the northsouth boundaries; that is,

$$
\begin{aligned}
T_{a}\left(\phi=\phi_{W}\right) & =T_{a}\left(\phi=\phi_{E}\right), \\
\frac{\partial T_{a}}{\partial \phi}\left(\phi=\phi_{W}\right) & =\frac{\partial T_{a}}{\partial \phi}\left(\phi=\phi_{E}\right), \text { and } \\
\theta & =-\theta_{S}, \theta_{N}: \frac{\partial T_{a}}{\partial \theta}=0 .
\end{aligned}
$$

The net downward heat flux into the ocean and land is given by

$$
\begin{aligned}
Q_{o a} & =\frac{\Sigma_{0}}{4} S(\theta)(1-\alpha) C_{0}-\mu\left(T-T_{a}\right) \quad \text { and } \\
Q_{l a} & =\frac{\Sigma_{0}}{4} S(\theta)(1-\alpha) C_{0}-\mu\left(T_{l}-T_{a}\right) .
\end{aligned}
$$

When the heat capacity of the land is assumed zero, then $Q_{l a}=0$ and the land temperature $T_{l}$ is computed directly from

$$
T_{l}=T_{a}+\frac{\Sigma_{0}}{4 \mu} S(\theta)(1-\alpha) C_{0} .
$$




\section{c. Numerical methods}

The equations are discretized in space using a secondorder accurate control volume discretization method on an equidistant $N \times M$ grid in the horizontal. A detailed setup of the model and its validation and resolution dependence for single-hemispheric basin flows has been discussed in Dijkstra et al. (2001). The vertical grid can be stretched toward the surface, using the function

$$
z_{k}=-D\left\{1-\frac{\tanh \left[q_{z}\left(\tilde{z}_{k}+D\right)\right]}{\tanh \left(q_{z} D\right)}\right\},
$$

where $k=0, \ldots, L$ indicates the vertical level, $q_{z}$ is a stretching factor, and the $\tilde{z}_{k}$ are equidistant, with $\tilde{z}_{0}=$ $-D$ and $\tilde{z}_{L}=0$.

The problem of computing steady-state solutions of the coupled ocean-atmosphere model leads, after discretization, to a set of nonlinear algebraic equations of the form

$$
\mathbf{F}(\mathbf{u}, \mathbf{p})=0 .
$$

Here $\mathbf{u}$ is a $d=6 \times N \times M \times(L+1)$-dimensional vector consisting of the unknowns at the gridpoints, $\mathbf{p}$ is the $p$-dimensional vector of parameters, and $\mathbf{F}$ is a nonlinear mapping from $R^{d} \rightarrow R^{d}$. The branches of steady states are determined using a pseudoarclength continuation technique; details are provided in Dijkstra (2000).

As can be readily seen from the steady equations (9), the salinity is determined up to an additive constant. Moreover, the pressure is also determined up to an additive constant. To calculate a steady-state solution of the system of Eq. (7), the equations are regularized (such that the Jacobian matrix is nonsingular at each regular point) by fixing the pressure at a particular point [in our case at the point $(N, M, L)]$. In addition, an integral condition for $S$ is substituted for the last equation from the salinity equation, such that salt is conserved exactly within the domain

$$
\frac{1}{\mathcal{V}} \int_{\mathcal{V}} S r_{0}^{2} \cos \theta d \phi d \theta d z=S_{0},
$$

where $\mathcal{V}$ is the total volume of the basin and $S_{0}$ is the reference salinity.

In the results below, many of the parameters will be fixed; these parameters are shown in Table 1. The values of the other parameters will be specified for each case below. The profile of the shortwave forcing $S(\theta)$ and the atmospheric eddy diffusivity $D(\theta)$ are shown in Fig. 1a. The freshwater forcing is prescribed as one of the two profiles $F_{S}$ in Fig. $1 \mathrm{~b}$ and the wind stress pattern $\tau^{\phi}$ is derived from a zonal average of the Trenberth et al. (1989) data values over the region (Fig. 1c), with $\tau^{\theta}$ $=0$. All of the results below are without the use of convective adjustment. The reason for this is that such a procedure introduces artificial local equilibria, which makes it very time consuming to compute a branch of
TABLE 1. Standard values of fixed dimensional parameters used in

\begin{tabular}{|c|c|c|c|}
\hline$\Omega=7.3 \times 10^{-5}$ & $\left(\mathrm{~s}^{-1}\right)$ & $r_{0}=6.4 \times 10^{6}$ & (m) \\
\hline$A_{V}=1.0 \times 10^{-3}$ & $\left(\mathrm{~m}^{2} \mathrm{~s}^{-1}\right)$ & $\rho_{0}=1.0 \times 10^{3}$ & $\left(\mathrm{~kg} \mathrm{~m}^{-3}\right)$ \\
\hline$\alpha_{T}=5.6 \times 10^{-5}$ & $\left(\mathrm{~K}^{-1}\right)$ & $g=9.8$ & $\left(\mathrm{~m} \mathrm{~s}^{-2}\right)$ \\
\hline$K_{H}=1.0 \times 10^{3}$ & $\left(\mathrm{~m}^{2} \mathrm{~s}^{-1}\right)$ & $K_{V}=1.0 \times 10^{-4}$ & $\left(\mathrm{~m}^{2} \mathrm{~s}^{-1}\right)$ \\
\hline$C_{p o}=4.2 \times 10^{3}$ & $\left(\mathrm{~J} \mathrm{~kg} \mathrm{~K}^{-1}\right)$ & $\alpha_{S}=7.9 \times 10^{-5}$ & $\left(\mathrm{~m}^{2} \mathrm{~s}^{-1}\right)$ \\
\hline$\Sigma_{0}=1.36 \times 10^{3}$ & $\left(\mathrm{~W} \mathrm{~m}^{-2}\right)$ & $D_{0}=1.5 \times 10^{6}$ & $\left(\mathrm{~m}^{2} \mathrm{~s}^{-1}\right)$ \\
\hline$\rho_{a}=1.25$ & $\left(\mathrm{~kg} \mathrm{~m}^{-3}\right)$ & $H_{a}=8.4 \times 10^{3}$ & $(\mathrm{~m})$ \\
\hline$C_{p a}=1.0 \times 10^{3}$ & $\left(\mathrm{~J} \mathrm{~kg} \mathrm{~K}^{-1}\right)$ & $C_{0}=0.5$ & - \\
\hline$B=1.5$ & $\left(\mathrm{~W} \mathrm{~m} \mathrm{~m}^{-2} \mathrm{~K}^{-1}\right)$ & $A=216$ & $\left(\mathrm{~W} \mathrm{m^{-2 }}\right)$ \\
\hline$\mu_{o a}=4.0$ & $\left(\mathrm{~W} \mathrm{~m} \mathrm{~m}^{-2} \mathrm{~K}^{-1}\right)$ & $\alpha=0.3$ & 一 \\
\hline$T_{0}=288.0$ & $(\mathrm{~K})$ & $S_{0}=35$ & 一 \\
\hline
\end{tabular}
the numerical calculations. The values of $\tau_{0}, H_{m}, D$, and $A_{H}$ will be specified for each result below.

equilibria in parameter space (Vellinga 1998). In the density distributions of the solutions below, there are consequently small regions for which solutions are statically unstable. However, results in a single-hemispheric basin indicate that it does not modify the branches of the large-scale flow solutions (Dijkstra et al. 2001).

\section{Idealized cases}

In this section, we want to illustrate the effect of the presence of a representation of the ACC on the equilibria of the Atlantic overturning circulation in its simplest form, using the three-dimensional coupled model. The impact of other equatorial asymmetry mechanisms, asymmetric freshwater flux, and the effect via coupling of a longitudinally smaller extent of the northern Atlantic in comparison with the Southern Ocean, are already known qualitatively from a two-dimensional model (Dijkstra and Neelin 2000); we focus on ACC impacts here before turning to the fully asymmetric case in section 4. Two cases of freshwater flux will be studied, both of which are symmetric about the equator and have no zonal structure. The two cases are used because the latitudinal dependence of the freshwater flux is important to an aspect of the bifurcation diagram that turns out to be crucial to the hysteresis behavior in the fully asymmetric case. In addition to a freshwater flux that is similar to a symmetrized version of that observed, using a simpler freshwater flux with a single, equatorial maximum allows us to discuss the origins of this feature in symmetric and small-asymmetry cases.

We begin by investigating the effect of the presence of a Southern Ocean (which is relatively narrow in latitudinal width) relative to a sector ocean case. The impact of this on the asymmetry is small enough that it can be considered an imperfection to the symmetric bifurcation diagram. To that end, first an equatorially symmetric sector domain is considered, with $\phi_{W}=282$, $\phi_{E}=354, \theta_{S}=-70$, and $\theta_{N}=70$, in which at the east-west boundaries a sector of $4^{\circ}$ is covered by land. The basin has a flat bottom and its depth $D$ is $4 \mathrm{~km}$; we will refer to this as the "closed" equatorially symmetric configuration. Subsequently, a possibility for an 

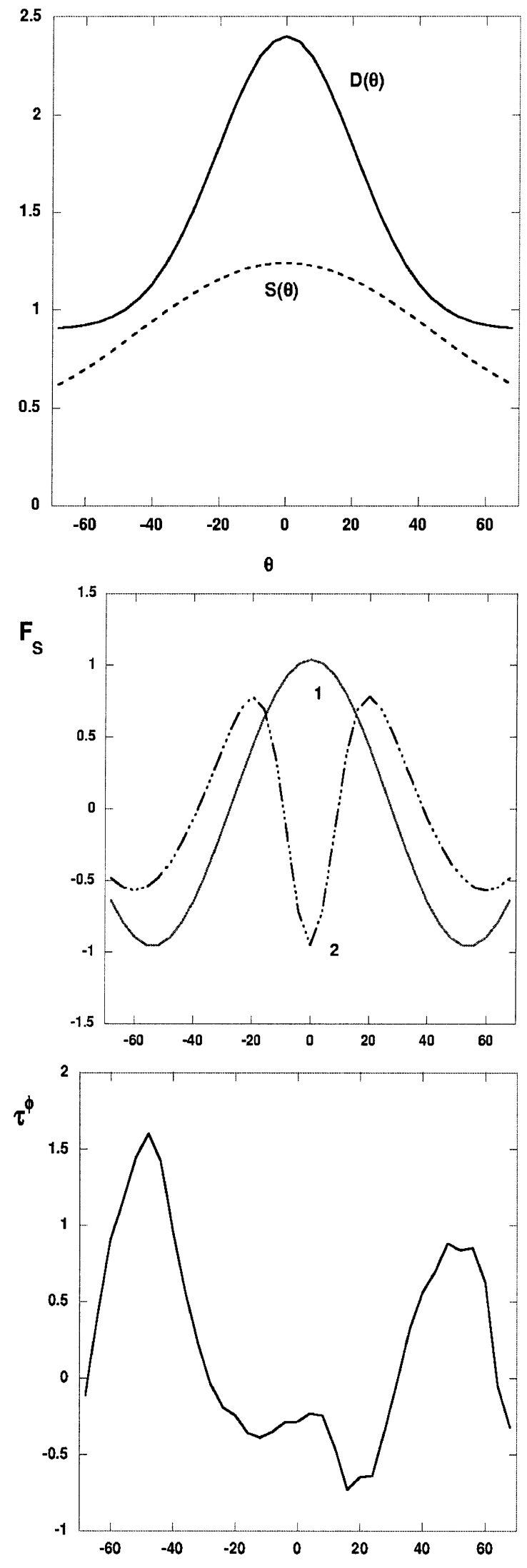

(a)

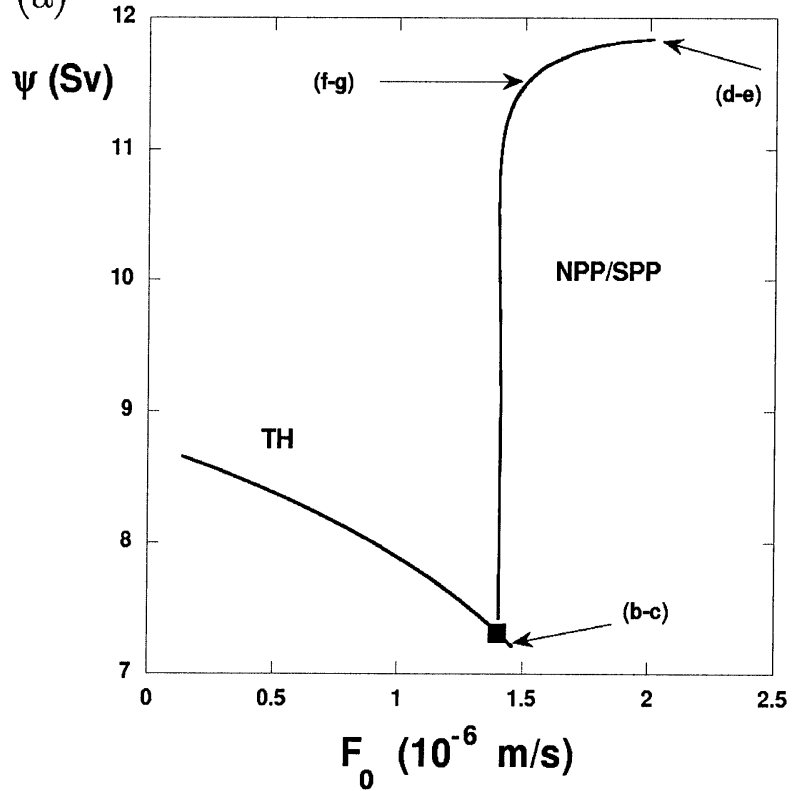

FIG. 2. (a) Bifurcation diagram for the closed configuration as a function of $F_{0}$, a measure of the strength of the freshwater flux. The maximum of the absolute value of the meridional overturning streamfunction $\psi(\mathrm{Sv})$ is used as a measure of the solution. For this symmetric case, northern and southern sinking branches overlay one another. (b), (d), and (f) Patterns of the overturning streamfunction (contours in Sverdrups) as a function of latitude and depth at the points labeled with the corresponding letters in (a). (c), (e), and (g) Surface horizontal velocity field (arrows) and vertical velocity at the base of the uppermost level (contours) as a function of longitude and latitude at labeled points in (a). Dark regions show outlines of idealized continents.

ACC to develop is created by removing part of the continents to allow for an open southern channel of $16^{\circ}$ latitudinal width. Periodic boundary conditions are applied in zonal direction to the southern channel; this is referred to as the "open" configuration. First, the wind stress is set to zero so that the implications of the oceanbasin geometry alone may be seen.

To ease our task to "deform" bifurcation diagrams from the "closed" to the "open" case, we take a relatively large horizontal friction $A_{H}=5.0 \times 10^{8} \mathrm{~m}^{2} \mathrm{~s}^{-1}$, a grid of $4^{\circ}$ horizontal resolution, and 16 vertical equidistant layers (a grid of $18 \times 35 \times 16$ ) such that $H_{m}$ $=250 \mathrm{~m}$. A bifurcation diagram for the closed case, and for parameters as in Table 1, is shown in Fig. 2a. The maximum of the meridional overturning streamfunction $\left(\mathrm{Sv} \equiv 10^{6} \mathrm{~m}^{3} \mathrm{~s}^{-1}\right)$ is plotted versus the freshwater-flux strength $F_{0}\left(10^{-6} \mathrm{~m} \mathrm{~s}^{-1}\right)$ for the freshwaterflux profile $F_{S}^{1}$. Note that a value $F_{0}=10^{-6} \mathrm{~m} \mathrm{~s}^{-1}$

FIG. 1. Dimensionless profiles of the forcing functions. (a) Shortwave radiative flux $S(\theta)$ and the eddy diffusivity in the atmosphere $D(\theta)$. (b) Two types of freshwater flux shapes $F_{S}^{i}$ (with $i=1,2$ ) used in the computations. (c) Profile of the wind stress forcing pattern $\tau^{\phi}$. 
(b)

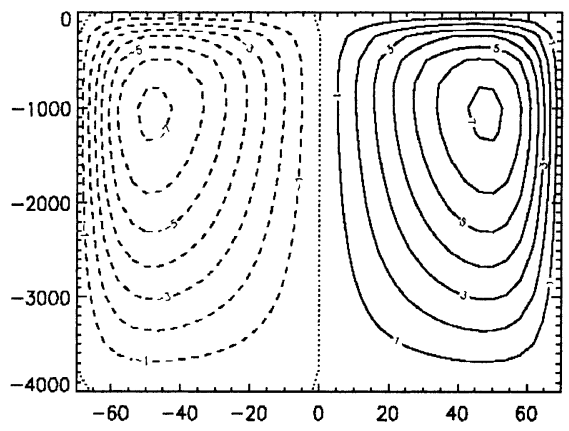

(d)

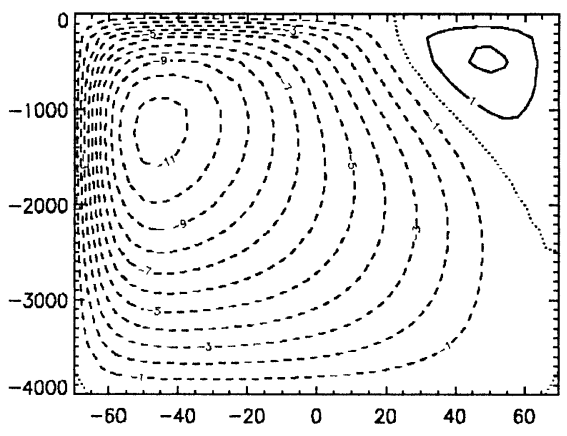

(f)

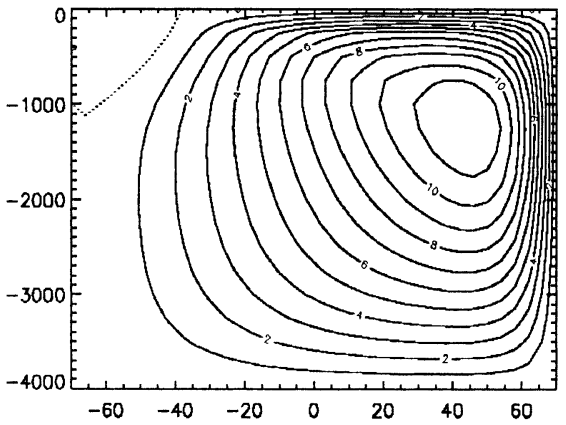

(c)

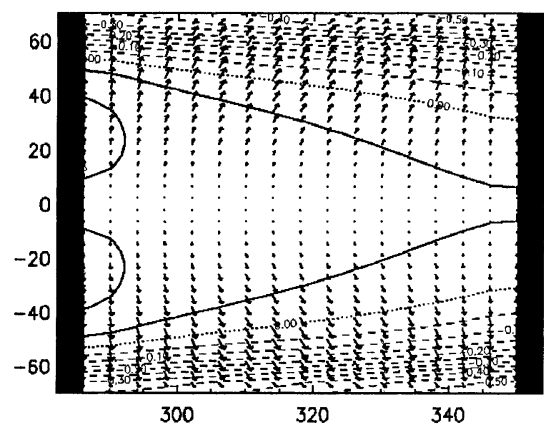

(e)

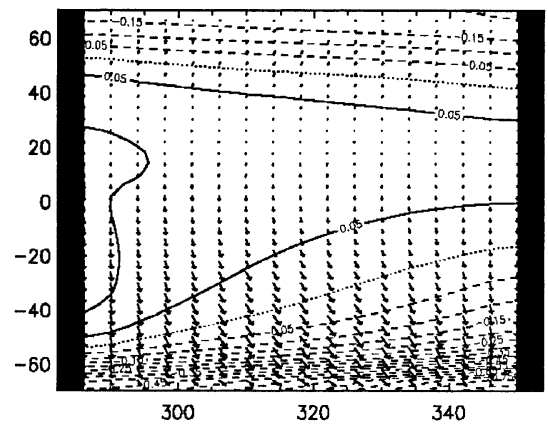

(g)

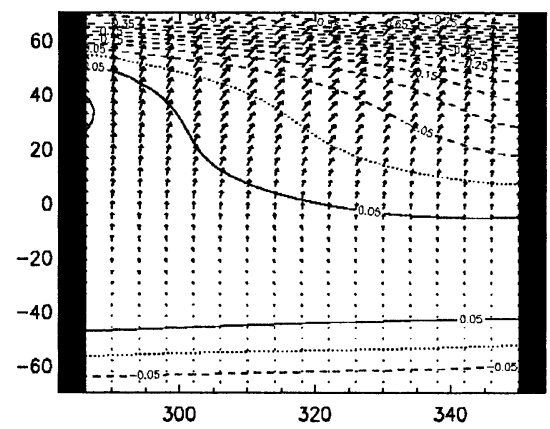

FIG. 2. (Continued)

corresponds to an actual value of $P-E=74 \mathrm{~mm}$ month $^{-1}$. Patterns of the overturning and the surface velocity field, with arrows indicating the horizontal velocity field and contours the vertical vertical velocity at labeled points in Fig. 2a, are plotted in Figs. 2b-f.

The symmetric thermally driven solution (Figs. 2b,c), also referred to as TH state (Thual and McWilliams 1992), becomes unstable through a pitchfork bifurcation near $F_{0}=1.40 \times 10^{-6} \mathrm{~m} \mathrm{~s}^{-1}$ and two asymmetric solutions appear. By symmetry, these have the same overturning strength and hence show up as a single curve in Fig. 2a. The streamfunction and surface velocity patterns show the Pole-to-Pole solutions, also referred to as southern sinking (SPP: Figs. 2d,e) and northern sinking (NPP: Figs. 2f,g) solutions, known from two-dimensional models (Thual and McWilliams 1992). The pitchfork bifurcation is supercritical since both asymmetric solutions occur at $F_{0}$ values larger than that at bifurcation. This bifurcation structure is very similar to that in the two-dimensional case as is expected from the results in Weijer and Dijkstra (2001). Note that, because of the large lateral friction, the sinking regions are located on the northern and southern boundaries; with much smaller lateral friction, the sinking regions move to the eastern boundary (Weijer and Dijkstra 2001).

The procedure to obtain the solution for the open configuration is as follows. A very large linear friction is applied to the part of the "missing" continental boundaries in the open configuration. In this way, the solutions for the closed configuration can be used as starting solutions for the open configuration and the branches are traced to those for which the linear friction coefficient is zero. For the freshwater flux forcing $F_{S}^{1}$, the bifurcation diagram for the open configuration is plotted in Fig. 3a as the solid and dashed curves, while 
the branches for the closed case (Fig. 2a) are replotted as the dotted curves. In the open configuration, the pitchfork bifurcation is no longer present and two disconnected branches appear; a so-called imperfect pitchfork bifurcation has occurred. The NPP branch of the closed configuration has connected to the small $F_{0}$ part of the $\mathrm{TH}$ branch, whereas the SPP branch has connected to the large $F_{0}$ part of the TH branch. Thus the connected branch (NPP/TH) is the northern sinking solution, whereas the isolated branch (SPP/TH) is the southern sinking solution. The latter exists only for $F_{0}$ values larger than those at the saddle-node bifurcation at $F_{0}=$ $1.42 \times 10^{-6} \mathrm{~m} \mathrm{~s}^{-1}$.

For the northern sinking solution (Figs. 3b,c), the flow through the southern channel is nearly absent (for this case with no wind forcing). However, for the southern sinking solution a relatively strong zonal flow appears through the channel (Figs. 3d,e). The solution on the lower SPP/TH branch (Figs. 3f,g) also displays substantial flow through the channel, causing a relatively large north/south asymmetry. Meridional pressure gradients in the open channel tend to drive a zonal flow, so this is a symptom of the different dynamics required to drive an overturning cell across the open channel. Additional discussion of the relation of these physical mechanisms to the bifurcation diagram is deferred to section 5.

With a more realistic freshwater-flux forcing (the profile $F_{S}^{2}$ in Fig. 1b), the bifurcation diagrams for the closed and open configuration change into those shown in Fig. 4a. The effect of the more realistic freshwater flux profile is a shift of the branches toward larger $F_{0}$. The pitchfork bifurcation in the closed case is now subcritical since the asymmetric solutions near it occur for smaller values of $F_{0}$. The subcriticality has an immediate impact on the imperfection when the southern channel is opened. Both NPP/TH and SPP/TH branches have associated hysteresis behavior over an interval in $F_{0}$ slightly smaller than that of the subcritical branch of the closed case (Fig. 4a). The lower branch of the SPP/ TH turns to higher values of $F_{0}$ through another saddlenode bifurcation and eventually form an isolated branch, but this is not shown here, since focus is on the behavior near the pitchfork bifurcation. The sensitivity of the type of pitchfork bifurcation to the shape of the freshwater flux is similar to that in two-dimensional model results (Dijkstra and Neelin 2000).

In the pattern of the NPP/TH solution (Figs. 4b,c), the effect of the midlatitude extrema of the freshwater flux can be seen as the additional near-surface, southern midlatitude overturning cell. Such a midlatitude cell can also be found in the solution of the upper branch of the SPP/TH branch (Figs. 4d,e), while the solution on the lower SPP/TH branch is nearly a TH solution with a slightly stronger southern cell (Figs. 4f,g). As in the previous case, the strong southern sinking solution has substantial transport through the southern channel (Fig. $4 \mathrm{e})$ while the flows on the NPP/TH branch have little.

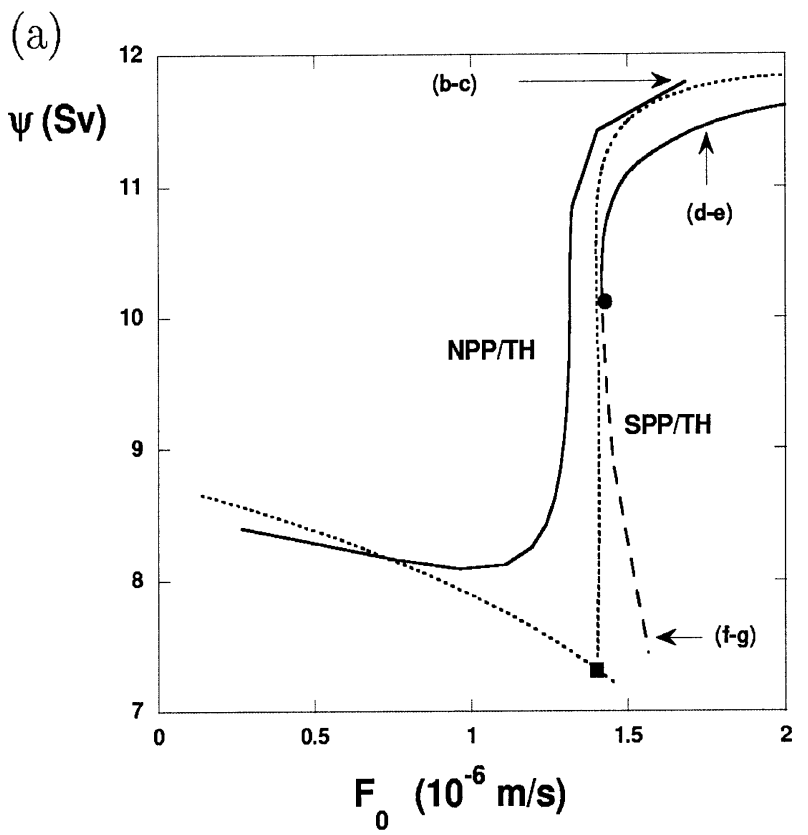

FIG. 3. (a) Bifurcation diagram for the open (solid-dashed) configuration and for comparison also those for the closed configuration (dotted), for the freshwater flux forcing $F_{S}^{1}$. (b)-(g) Patterns of the overturning streamfunction and surface velocity field at locations labeled in (a). Conventions are as in Fig. 2.

For the open configuration and the freshwater flux profiles $F_{S}^{2}$, we now investigate how the slight preference for the northern sinking solution is affected by the addition of wind stress forcing. The wind stress profile as in Fig. 1c is applied and both NPP/TH and SPP/TH branches in Fig. 4a are followed when the amplitude is increased from $\tau=0.0 \mathrm{~Pa}$ to realistic strength $\tau_{0}=0.1$ $\mathrm{Pa}$. The NPP/TH branch exists for all values of $\tau_{0}$ and the branch for $\tau_{0}=0.1 \mathrm{~Pa}$ is plotted as the solid curve in Fig. 5a. The wind stress has an impact on the hysteresis within the northern sinking branch as the hysteresis interval is now very small (the saddle-node bifurcations are located at $F_{0}=2.88 \times 10^{-6} \mathrm{~m} \mathrm{~s}^{-1}$ and $F_{0}=2.90$ $\times 10^{-6} \mathrm{~m} \mathrm{~s}^{-1}$ ). The solution in Figs. $5 \mathrm{~b}$ and $5 \mathrm{c}$ indicates that the wind has lead to only a small effect on the transport through the southern channel because of the relatively large horizontal viscosity in the model. However, it turns out that the wind stress has a profound influence on the existence of the $\mathrm{SPP} / \mathrm{TH}$ solution. The isolated branch for $\tau_{0}=0.04 \mathrm{~Pa}$ is shown as the dotted curve in Fig. 5a. This branch cannot be followed to the case of full wind stress strength $\left(\tau_{0}=0.1 \mathrm{~Pa}\right)$ because it becomes an isola. There is an upper limit of existence of this branch in $F_{0}$, with a strong southern sinking solution (Figs. 5d,e) and lower limit in $F_{0}$ with a weak southern sinking solution (Figs. 5f,g). The former has a substantial transport through the southern channel.

In addition, the wind stress reduces the interval of the existence of multiple equilibria and this interval has also shifted to slightly smaller values of $F_{0}$. When the effect 
(b)

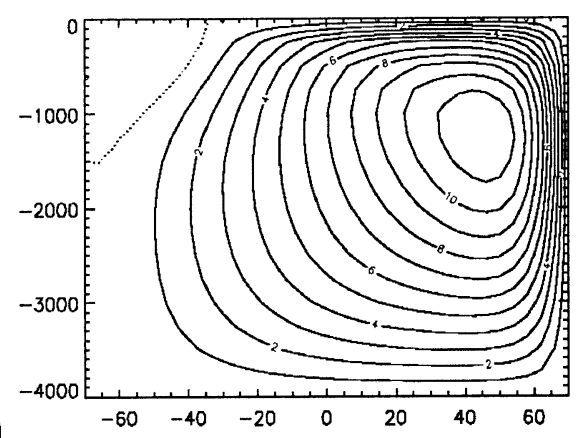

(d)

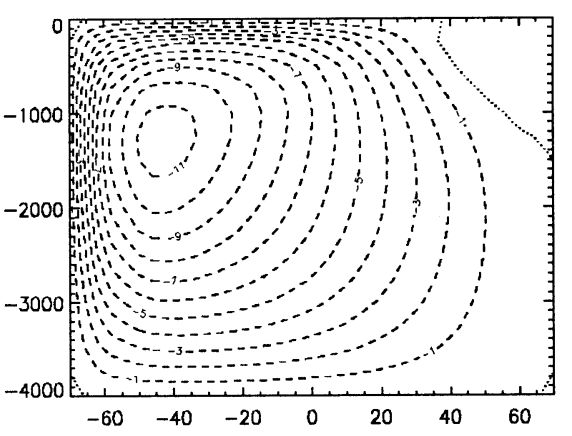

(f)

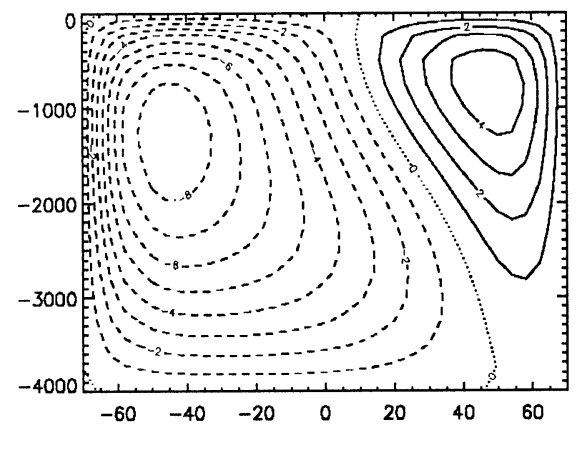

(c)

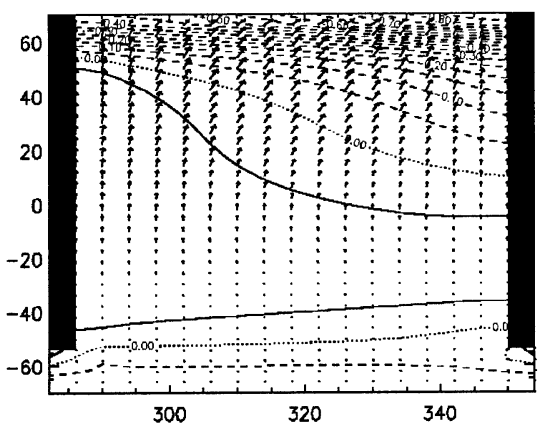

(e)

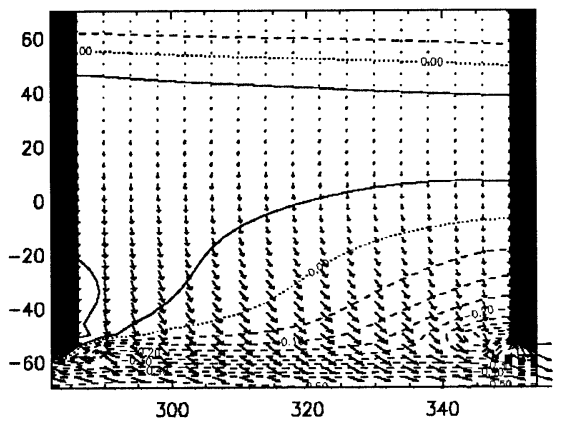

(g)

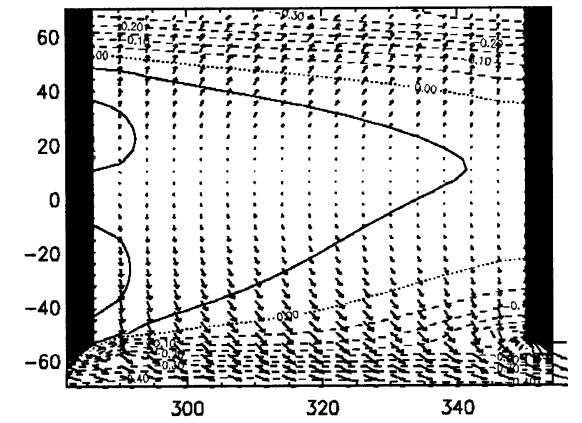

FIG. 3. (Continued)

of wind stress is considered in closed ocean-only configurations, with an even more idealized shape, its effect is much larger since a wide area of hysteresis opens in that case (Weijer and Dijkstra 2001). One reason for this difference is that, in the coupled model, the effect of the wind stress is smaller than in the ocean-only model (Rahmstorf and England 1997). In the north, the ocean water can be cooled more than in the uncoupled case, leading to a stronger buoyancy control over the flow.

\section{Atlantic geometry}

Within a sector domain, with $\phi_{W}=-85, \phi_{E}=20$, $\theta_{S}=-70$, and $\theta_{N}=70$, continental geometry and bathymetry (as derived from the ETOPO-10 dataset, online at http://www.ngdc.noaa.gov/mgg/global/) were interpolated on a $40 \times 30 \times 14$ grid. The vertical levels are nonequidistant with stretching factor $q_{z}=2.0$ in (6); as a consequence $H_{m}=54 \mathrm{~m}$ for this case. To obtain a suitable pattern of the freshwater-flux forcing, steady states were first determined using the zonally averaged Levitus et al. (1994) profile of surface salinity (Fig. 6a) using a restoring boundary condition. Slightly different parameter values of the mixing parameters than in Table 1 were used: $K_{H}=$ $2 \times 10^{3} \mathrm{~m}^{2} \mathrm{~s}^{-1}$ and $K_{V}=2 \times 10^{-4} \mathrm{~m}^{2} \mathrm{~s}^{-1}$. Following the practice of many GCM studies (Tziperman 2000), the freshwater flux is then diagnosed. The restoring solution is equatorially asymmetric, with a stronger northern sinking cell (Fig. 6b) and the freshwater flux $(P-E)$ maintaining this circulation is shown in Fig. 6c. It has similar features as the idealized profile $-F_{S}^{2}$ with net evaporation at midlatitudes and net precipitation at high latitudes. There is a small net precipitation zone just north of the equator; the maximum dimensional amplitudes of the freshwater flux is $150 \mathrm{~mm}$ month $^{-1}$.

The bifurcation diagram is then computed using this 
freshwater flux, the profile of the shortwave forcing $S(\theta)$, the atmospheric eddy diffusivity $D(\theta)$, the zonal wind stress shape as shown in Fig. 1 for standard values of the parameters in Table 1, and the smoothed Atlantic geometry and bottom topography. All mechanisms for equatorial asymmetry are thus present for this case. In principle, we could attempt to dissect the contribution of each source of asymmetry by symmetrizing each in turn, but the impact of continental configuration is sufficiently large that the diagrams tend to be qualitatively similar and the computations for this realistic domain are more costly. Since the asymmetry mechanisms all favor similar behavior, we can simply verify the relation of the bifurcation diagram in this realistic case to that obtained in the asymmetric idealized case.

The bifurcation diagram for the realistic Atlantic case, with $A_{H}=1.6 \times 10^{7} \mathrm{~m}^{2} \mathrm{~s}^{-1}, \tau_{0}=0.1 \mathrm{~Pa}$, and all other parameters as in Table 1 are shown in Fig. 7. As in earlier bifurcation diagrams, the strength of the northern overturning cell is plotted (in Sverdrups) versus the strength of the freshwater flux $\gamma$; the maximum amplitude of the latter is $\gamma \times 150 \mathrm{~mm}$ month $^{-1}$. The steady solution for $\gamma=1.0$ differs slightly from the solution with the surface salinity profile of Fig. 6a because of the slightly different parameter values used in the restoring case. For this case, only one branch is found that displays a small region of multiple equilibria. As can be deduced from the idealized case, this is the deformation of the NPP/TH branch to the realistic domain. The southern sinking solutions cannot be found as steady states for this configuration; they had already disappeared in the idealized case under realistic wind forcing. Because in principle these equilibria could reappear, an additional continuation path was used to try to identify connections, if any, to a possible southern sinking branch. A parameter was introduced to set the latitudinal variation of the insolation to zero, with realistic freshwater forcing. The solution thus began from a saline-driven branch, analogous to that found in simpler model bifurcation studies at high freshwater-flux forcing. The saline branch was then followed up to realistic thermal forcing at the standard insolation. It connected to the same branch shown above. This provides quite strong evidence that the SPP/TH branch, if it exists at all, must be an isola such as was seen in the idealized case for intermediate wind forcing (or totally absent). Based on the lack of encounters with the branch in a number of other idealized geometry experiments and the demonstrated disappearance of the isola in the previous section, we think that it is unlikely that this branch exists for realistic asymmetry.

Patterns of the streamfunction and surface circulation are shown in Fig. 8 for the solutions at selected values of $\gamma$ on the branch in Fig. 7. At small values of $\gamma$, the overturning steamfunction is somewhat asymmetric (Figs. 8a,b) but still bears much relation to a two-cell symmetric TH branch; in correspondence with the results in Dijkstra and Neelin (2000), we call this the ATH (a)

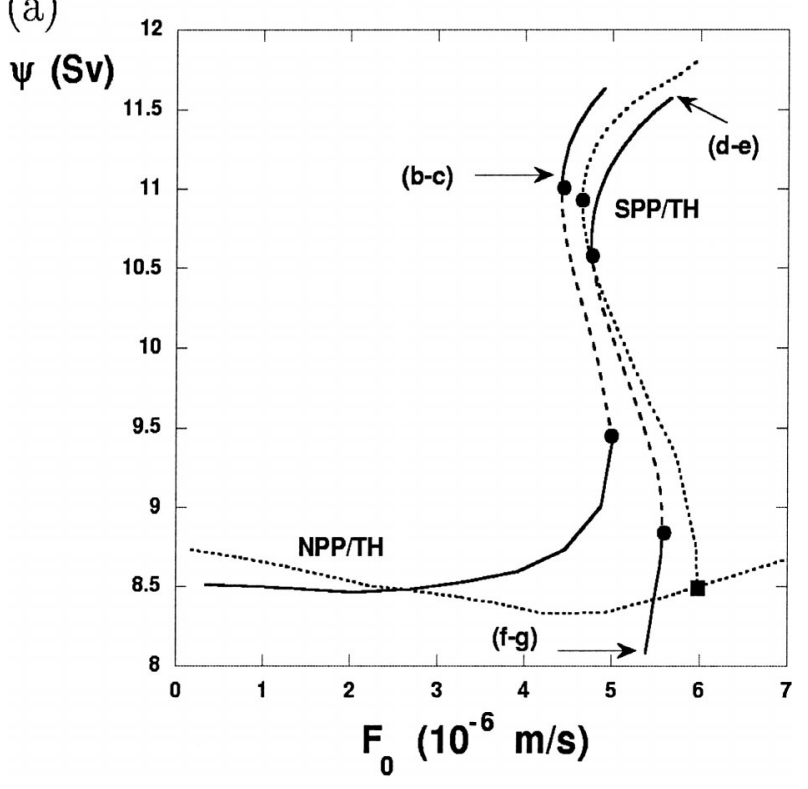

FIG. 4. (a) Bifurcation diagram for the open configuration for the freshwater flux forcing $F_{S}^{2}$ and for comparison also those for the closed configuration (dotted), with (b)-(g) patterns of the overturning streamfunction and surface velocity field at locations labeled in (a). Conventions are as in Fig. 2.

solution branch (Fig. 7). At larger values of $\gamma$, the salinity feedback becomes more and more important, as is easily seen by comparing the case for $\gamma=0.62$ with the others (Figs. $8 \mathrm{c}-\mathrm{h}$ ). The stable solutions within the multiple equilibria region differ slightly in the strength of the southern sinking cell (Figs. 8c,d vs Figs. 8e,f), while the latter completely disappears at large $\gamma$ (Figs. $8 \mathrm{~g}, \mathrm{~h})$. For all solutions, there is substantial flow through the southern channel.

\section{Physical mechanisms}

We turn now to mechanisms underlying the results above. Questions of interest include (i) why is the northern sinking solution preferred in the open configuration, (ii) why does the wind on the ACC enhance this, (iii) why does the southern sinking branch form an isola and disappear with increasing wind forcing, (iv) what is the role of the salt advection feedback in the unique-state regime, and (v) what is the difference between the cases with and without hysteresis? The above questions can be discussed in the context of the idealized geometry case of section 3, although they apply to the realistic geometry case as well.

With regard to the preference for the northern sinking branch (question i), the starting point of the explanation is the closed case and the symmetric flows as in Fig. 2. In the southern part of the basin, the geostrophic response to the meridional buoyancy gradient is a strong zonal eastward flow. Because of the presence of the eastern solid boundary, a zonal pressure gradient is created that 
(b)

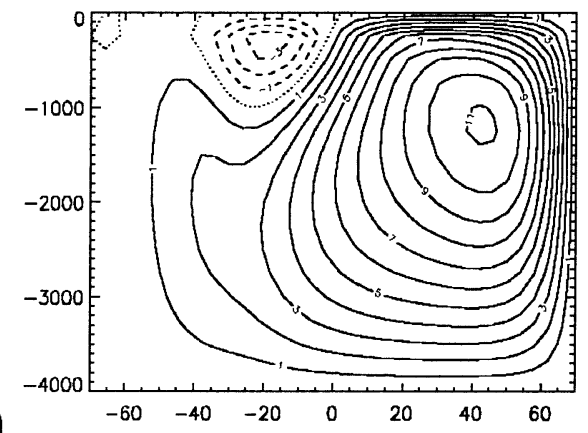

(d)

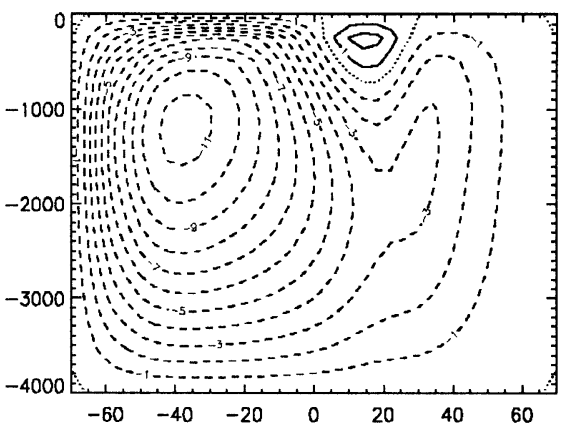

(f)

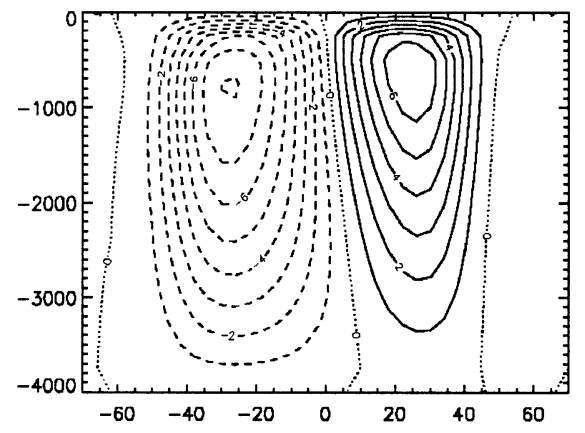

(c)

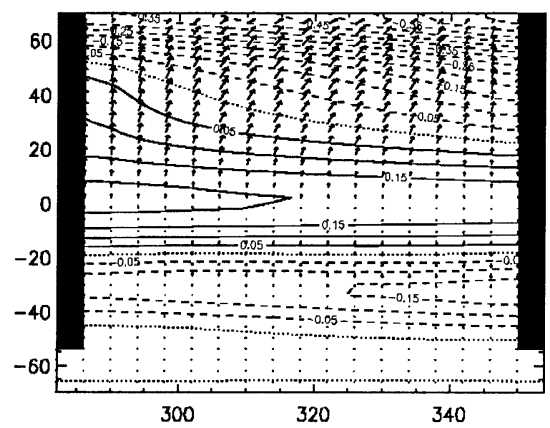

(e)
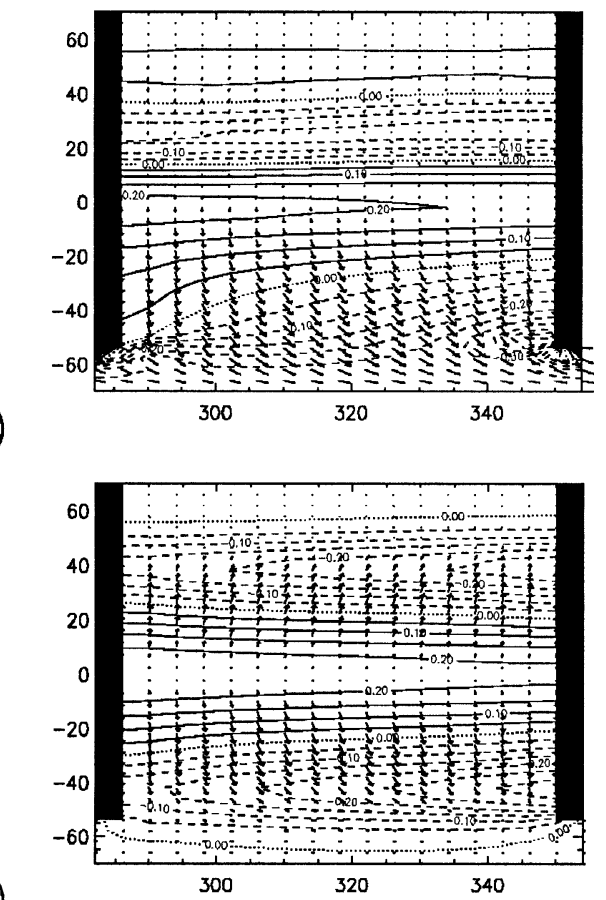

(g)

FIG. 4. (Continued)

drives a meridional flow. The same occurs in the northern part of the basin giving the symmetric TH overturning flow. When there is an open channel somewhere, with periodic boundary conditions, the zonal pressure gradient is effectively zero. In the open configuration, the zonal pressure gradient becomes small when approaching the zonal channel from the north. The presence of the boundary is essential for sinking to occur because one needs the boundary layers to break geostrophy (Spall and Pickart 2001). Consequently, the resulting meridional flow is weaker than that in the closed northern part of the boundary. To phrase this in another way, if one were to place a density anomaly near the northern boundary or near the southern boundary, the resulting surface meridional flow would be weaker across the southern ocean than in the northern basin simply due to the lack of a zonal boundary against which to maintain a zonal pressure gradient. This asymmetry consequently slightly favors the northern sinking branch.
In terms of its implication for the equilibrium solution branches, the favoring of the northern sinking branch produces an imperfection much like those noted for other physical mechanisms as considered in Dijkstra and Neelin (2000). In the latter paper, a parameter $\sigma$ was used to measure the strength of the freshwater flux. We will use both parameters below in referring to the results, keeping in mind that $F_{0}$ in the present paper is just a constant factor times $\sigma$.

In the symmetric case (Fig. 9a), both asymmetric branches can only be maintained by the salt advection feedback if the freshwater forcing is strong enough. In the open case, it is relatively easier to establish a northern sinking branch because the feedback of meridional flow for a given density change is stronger. The northern sinking branch thus connects to the thermally dominated branch and exists for every $\sigma$ value as shown for the upper branch in Fig. 9b, becoming more and more asymmetric for larger $\sigma$. Because the salt advection feedback 
is weakened by the asymmetry for the southern sinking branch (lower branch in Fig. 9b), it shifts toward larger values of $\sigma$, that is, larger than that at the pitchfork bifurcation in the closed case. It thus is constrained to connect to the unstable thermal branch, resulting in the saddle-node bifurcation in Fig. 3. The physical arguments favoring the northern sinking branch are the same whether the pitchfork bifurcation for the symmetric case is supercritical (as for the simplified freshwater flux $F_{S}^{1}$, dotted curves of Fig. 3) or subcritical (as in the more realistic freshwater flux $F_{S}^{2}$, dotted curves of Fig. 4). However, in the latter case, the northern sinking branch has a hysteresis bifurcation which at larger asymmetry results in a regime with two stable equilibria, a strong and a weak northern sinking state.

The impact of the wind stress (question ii) may be seen from the comparison of Fig. 4 and Fig. 5 to impact the southern sinking branch much more strongly than the northern sinking branch. The onset of high overturning circulation in the northern sinking branch occurs at slightly smaller values of $F_{0}$ when the wind stress is present and there are some quantitative changes in the hysteresis bifurcation, but large qualitative changes occur in the southern branch. The physical mechanism can thus be discussed primarily in terms of factors disfavoring the salt advection feedback for the southern sinking branch. The most obvious factor is that the Ekman flow is northward over a substantial portion of the Southern Ocean. The addition of an Ekman contribution of the flow will reduce the advection of salinity into the southern sinking region at the surface, with respect to the no-wind case. This will oppose the salt advection feedback for southern sinking, shifting the bifurcation to higher $F_{0}$ values or, if the wind is strong enough, preventing it altogether. From the results in section 3, wind values weaker than observed are already sufficient to remove the southern sinking branch. Another impact of the wind, the tilting of isotherms in the Southern Ocean (Rahmstorf and England 1997) such that deep water can be more easily ventilated to the surface, might slightly assist the northern sinking branch but it is not obvious that it would necessarily disfavor southern sinking solutions.

With regard to question iii, why the southern sinking branch forms an isola that shrinks to one point and disappears with additional wind stress: parallel arguments can be made for both the left (low $F_{0}$ ) boundary of the isola on the bifurcation diagram and the right (high $F_{0}$ ) boundary. First, it is necessary to recall from other bifurcation studies (Dijkstra and Molemaker 1997) that in the symmetric case a second pitchfork bifurcation occurs at very high values of the freshwater forcing parameter that leads to a salinity-driven solution branch. The latter solutions may actually be relevant for understanding flows in the geological past (Bice and Marotzke 2001). In this regime, the NPP and SPP branches disappear since there is no longer sufficient trade-off between the two effects on density for the salt advection feedback to gen- (a)

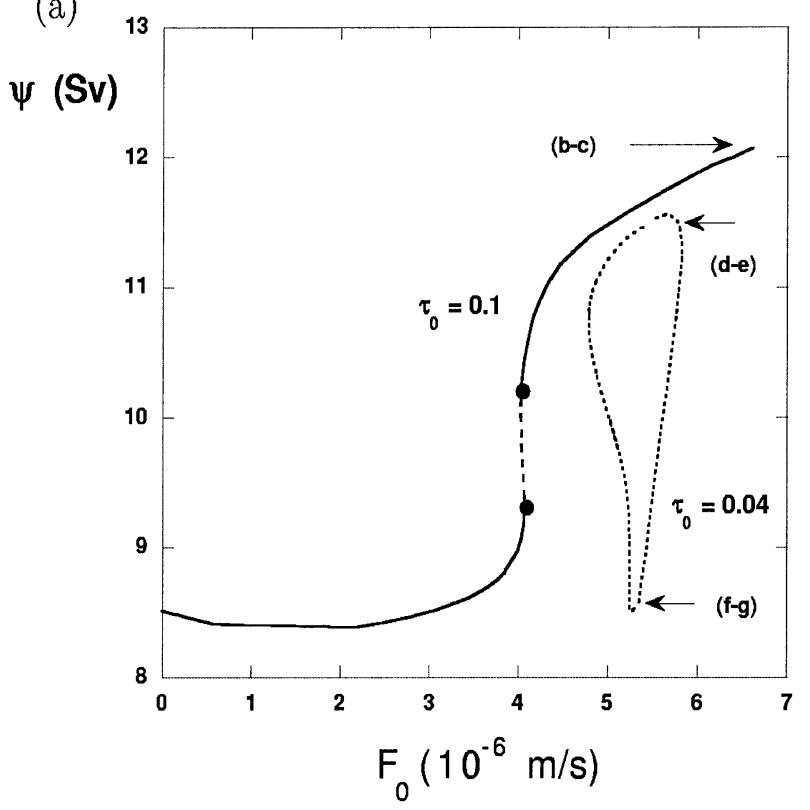

FIG. 5. (a) Bifurcation diagram for the open configuration and several values of the wind stress strength $\tau_{0}$, with (b)- $(\mathrm{g})$ patterns of the overturning streamfunction and surface velocity field at locations labeled in (a). Conventions are as in Fig. 2.

erate multiple equilibria. This is seen in the shaded part of the schematic diagram Fig. 9a, with $\sigma$ indicating the strength of the freshwater flux. We have not computed this part of the diagram explicitly in the three-dimensional coupled model and so are relying on qualitative correspondence to the two-dimensional models (Dijkstra and Molemaker 1997; Dijkstra and Neelin 2000) for this part of the diagram (some calculations that reduce thermal contrast instead of increasing $F_{0}$ suggest that this correspondence is reasonable).

Consider what happens as one reduces $\sigma$ from the salinity-dominated regime in case of a subcritical pitchfork bifurcation (upper panel of Fig. 9). With slight equatorial asymmetry, the pitchfork bifurcation must be broken so that the salinity dominated solution connects either to the northern sinking or the southern sinking branch. Maintaining either branch depends on the salt advection feedback. If any factor reduces the amount of southward transport that can be accomplished across the Southern Ocean for a given density anomaly near the southern boundary, as compared with the converse situation near the northern boundary, then the NPP solution will be favored and the saline-dominated branch will connect to the NPP branch. The SPP branch will appear at slightly lower $\sigma$ and must connect to the unstable symmetric branch. This immediately implies an isola for the southern branch, as illustrated in the middle panels of Fig. 9. The physical mechanisms favoring the NPP branch are presumably the same as discussed for the lower- $\sigma$ imperfection: first, the open Southern Ocean provides little east-west pressure gradient to balance 
(b)

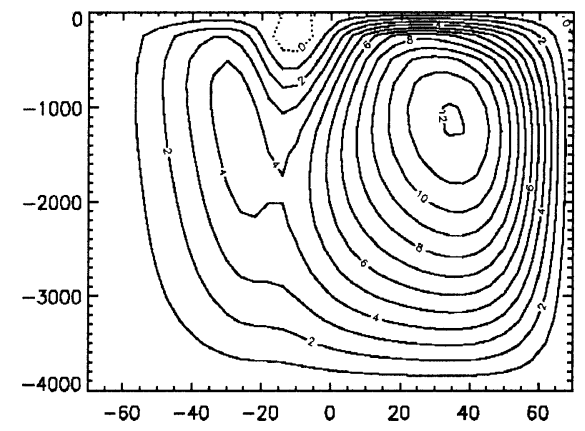

(d)

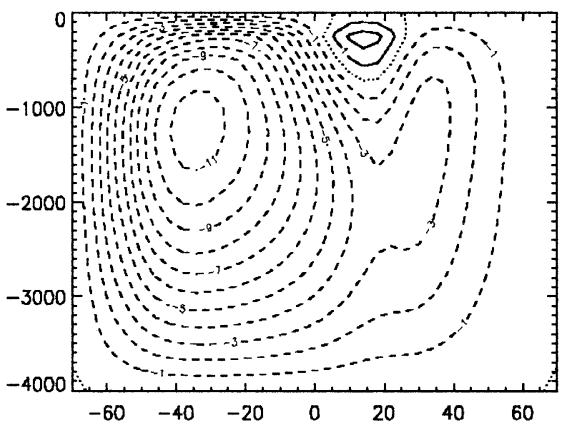

(f)

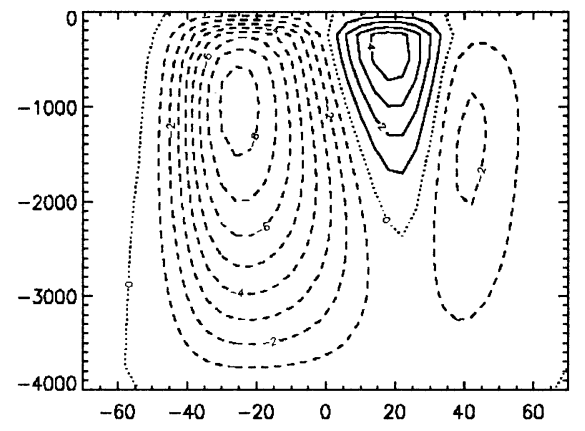

(c)
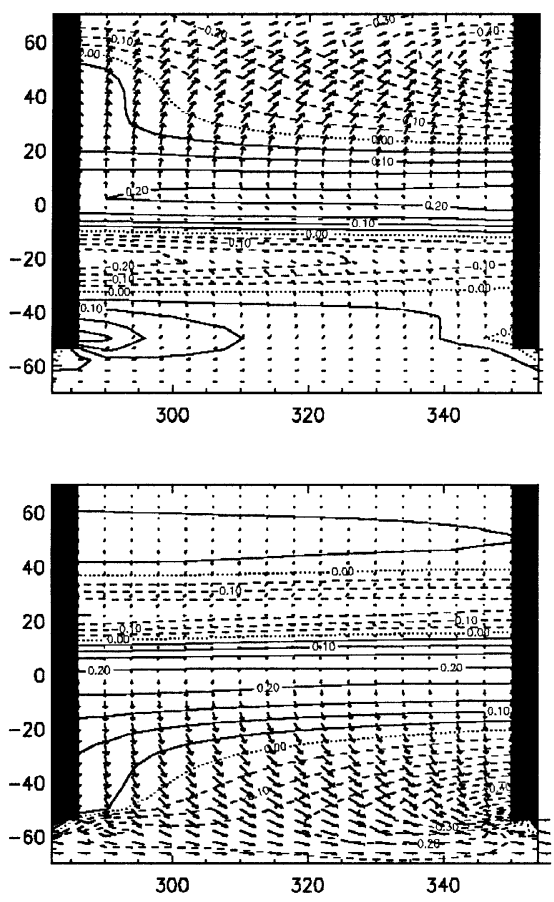

(e)

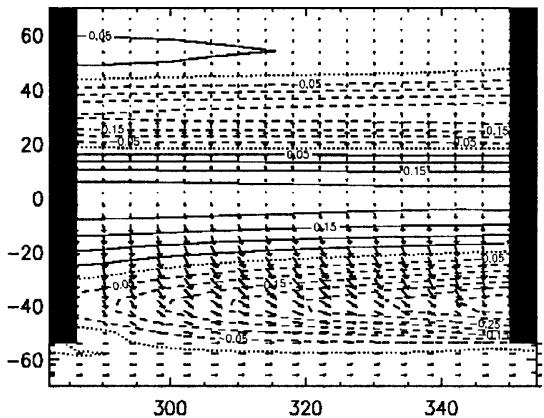

FIG. 5. (Continued)

meridional flow; second, the Ekman transport near what would be the southern sinking region is northward, opposing southward flow.

In the unique-state regime, the salt advection feedback is as important in enhancing the circulation in the strong northern sinking circulation, as it is in cases in which there are multiple equilibria (question iv). This may be seen by comparing the circulation for low $\sigma$ with the circulation at realistic $\sigma$ values in the unique-state regime. However, the other mechanisms that produce a tendency toward northern sinking due to equatorial asymmetry are sufficiently strong that the salt advection feedback in a southward surface flow perturbation is not sufficient to overcome these and establish another equilibrium state. Note that the Pole-to-Pole solutions are driven both by temperature and salinity, rather than driven by temperature and braked by salinity, as seems to be common wisdom (Stocker 2000). This can be concluded from the increase in overturning with increasing $F_{0}$, as seen in Figs. 5 and 7. Hence, the dynamics of these solutions cannot be captured by a single-hemispheric box model, such as the Stommel two-box model (Stommel 1961). It is compatible with a Pole-to-Pole two-box model (Rahmstorf 1996; Marotzke 2000), where it is assumed that the overturning is proportional to a north-south density difference. However, since the north-south density difference is dynamically related to the surface forcing, the latter model is not explanatory of the correct overturning relationship. Here, this relation is seen as a consequence of the symmetry breaking related to the pitchfork bifurcation.

With regard to question $\mathrm{v}$, the difference between the subcritical and supercritical pitchfork bifurcation has been identified as being associated with the source of the hysteresis behavior that is important to the surviving multiple equilibria under realistic asymmetry. Tradi- 


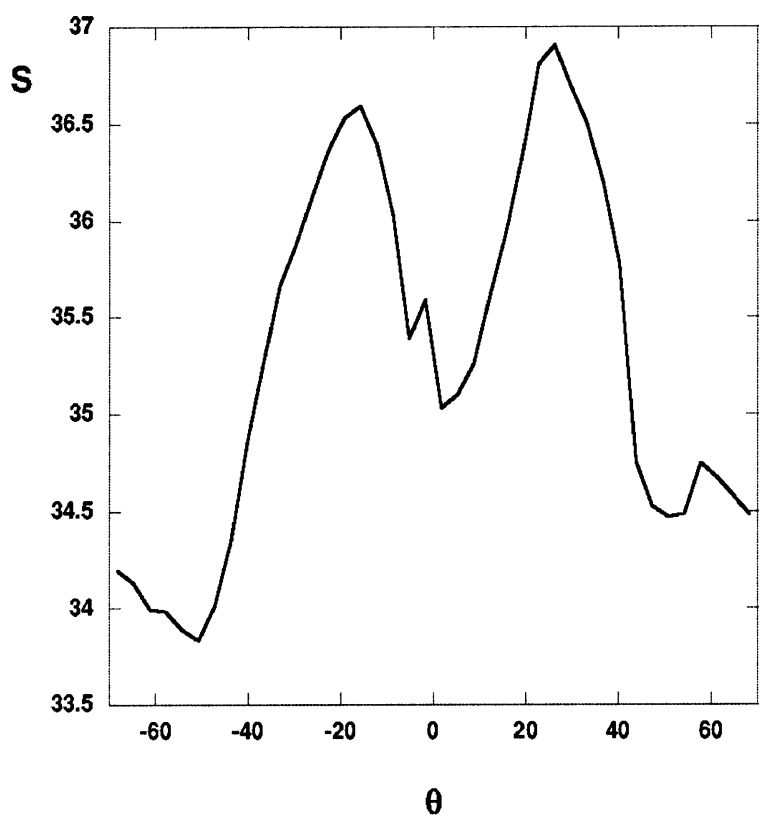

(a)

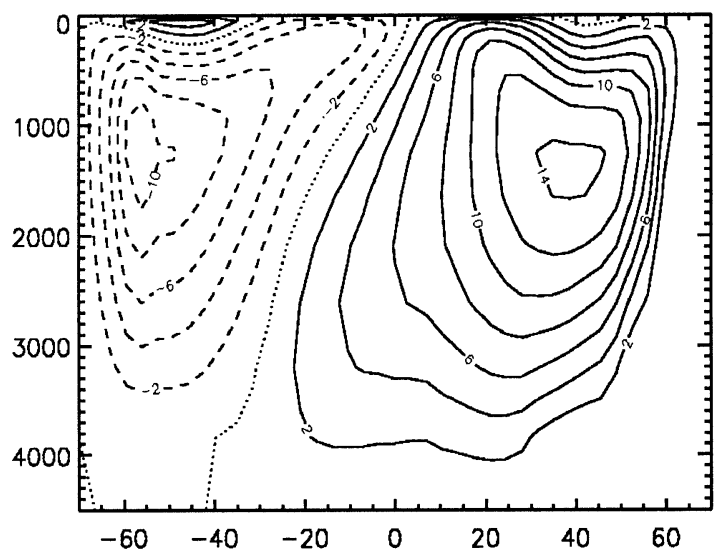

(b)

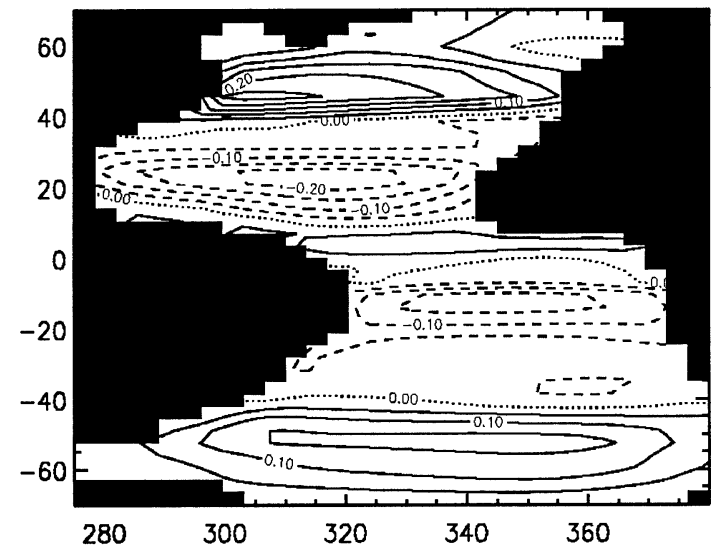

(c)

FIG. 6. (a) Profile of the zonally averaged profile of the Levitus et al. (1994) surface salinity. (b) Overturning streamfunction under restoring conditions for zonally averaged salinity. (c) The (dimensionless) freshwater flux pattern $-F_{S}(\phi, \theta)$ as used in the remainder of the computations in section 4. A value of 0.27 corresponds to a value of $P-E=150 \mathrm{~mm}$ month $^{-1}$.

tionally, a description of the salt advection feedback has been considered sufficient to explain the hysteresis bifurcation. However, the salt advection feedback is equally at work in both sub- and supercritical cases. A more subtle physical mechanism is required to explain the difference between supercritical and subcritical cases, that is, between the salt advection feedback for a case with maximal freshwater flux at the equator and the more realistic case with subtropical maxima. We do not have a definitive answer but we conjecture that the answer lies in the stabilization of the thermally dominated (TH) branch for the more realistic flux as outlined below. As seen in the results (Figs. 3 and 4), the position of the pitchfork bifurcation is shifted for different freshwater flux profiles. It is important to the physics that the subcritical bifurcation arises by shifting of the pitchfork bifurcation to much higher values of $\sigma$. The largeoverturning parts of the NPP and SPP solution branches also shift toward higher $\sigma$, but not by as much as the pitchfork bifurcation. Thus the impact of realistic freshwater flux is that the salt advection feedback is generally less destabilizing, but this impact is much greater for small-amplitude perturbations than for finite-amplitude departures from the symmetric state. We can thus rephrase the question as: why is the thermally dominated branch more stabilized to small perturbations than to 


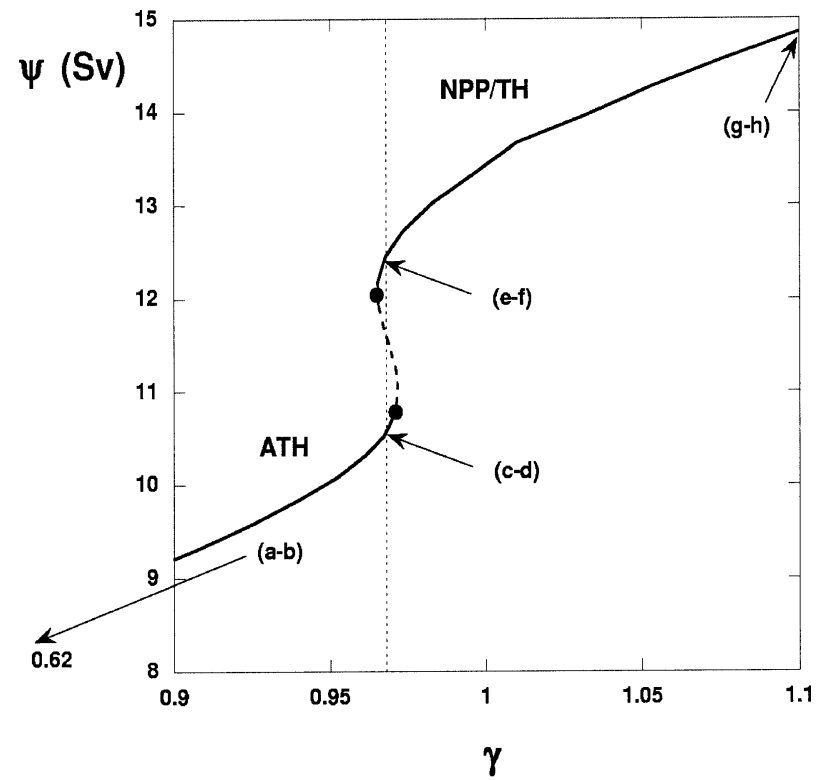

FIG. 7. Bifurcation diagram for the realistic Atlantic configuration. The parameter $\gamma$ is a measure of the strength of the freshwater flux (the amplitude of the freshwater flux is $\gamma \times 150 \mathrm{~mm} \mathrm{month}^{-1}$ ), with a pattern given in Fig. 6c.

large-amplitude perturbations when the freshwater flux changes from $F_{S}^{1}$ to $F_{S}^{2}$ ?

First, note that the essential ingredient of the salt advection feedback is the surface salinity, which tends to bear a strong resemblance to the freshwater flux; the flux $F_{S}^{1}$ will generate a salinity maximum at the Tropics, whereas $F_{S}^{2}$ will generate salinity maxima in the subtropics. A heuristic argument would be the following: suppose we have a salinity perturbation that is positive in the Northern Hemisphere and negative in the south. Such a bipolar structure is characteristic for the mode destabilizing the symmetric circulation at the pitchfork bifurcation (Weijer et al. 2001). It would generate an overturning anomaly with northward transport on shallow levels and southward flow at depth. In the case of the flux $F_{S}^{1}$, this perturbation would advect salty tropical waters to the Northern Hemisphere, thus enhancing the positive anomaly and making the positive salt advection feedback work. When there is a salinity minimum at the equator, as is the case for $F_{S}^{2}$, a weak overturning perturbation would advect relatively fresh water toward the Northern Hemisphere. This would attenuate the positive salinity anomaly and keep the perturbation from growing. Only when the overturning anomaly is strong enough to draw saltier subtropical water from the Southern Hemisphere across the equator, a positive feedback is established and the perturbation can grow. Hence, this would explain the stability changes to finite-amplitude perturbations. A more formal view of the physics of the difference between supercritical and subcritical pitchfork is presented in appendix B.

We note that this mechanism is not easy to capture in a Stommel model because the spatial structure of the freshwater flux is important. There could be other mechanisms of producing a subcritical pitchfork bifurcation by stabilizing the symmetric state to small perturbations over some range, while keeping the finite-amplitude salinity-feedback-dominated branch; however, the $F_{S}$ dependence appears a leading explanation.

\section{Summary and conclusions}

This is one of the first studies where the qualitative changes of the different equilibria of the three-dimensional thermohaline ocean circulation in parameter space have been studied using direct continuation techniques. The first aim of the study was to determine the qualitative effect of the presence of an open Southern Ocean and ACC-like flow on the bifurcation structure of the THC. These contribute additional mechanisms for latitudinal asymmetry about the equator in addition to the previously studied mechanisms listed in the introduction. An associated aim was to determine the relation between the structure of the equilibria found in two-dimensional coupled models in an idealized geometry (Dijkstra and Neelin 2000) and those in threedimensional models in a fairly realistic Atlantic configuration. In the course of this exploration, it was determined that the various asymmetries cooperate in favoring northern sinking solutions. As a result, cases with realistic asymmetry about the equator, including the open Southern Ocean and wind on the ACC, tend to have two important parameter regimes, termed the hysteresis regime and the unique-state regime respectively. A second aim of the study, perhaps more important in terms of climate implications, therefore became to determine the origin of these two regimes in terms of their relation to structures in the symmetric case and in terms of physical mechanism.

The transition between the idealized "closed" and "open" cases, examined in section 3, demonstrates the impact of opening the Southern Ocean. Even without wind, the open southern channel gives a preference for the northern sinking solution. The physical reason for this preference is that the southern passage reduces the zonal pressure gradient that can be sustained and thus decreases the meridional overturning circulation of the southern sinking solution. As this circulation is maintained by the salt advection feedback, a larger value of the freshwater forcing is needed for its existence. This is reflected in the presence of a saddle-node bifurcation that gives the lower limit of the southern sinking branch. As schematized in Figs. 9a,b, the opening of the southern channel introduces a modest imperfection that causes the TH branch to connect to the NPP branch. The SPP branch is connected through the saddle-node bifurcation to the unstable thermal branch and this configuration is shifted to larger freshwater forcing.

In the idealized open case, the effect of including wind stress, driving an ACC and associated Ekman 
(a)

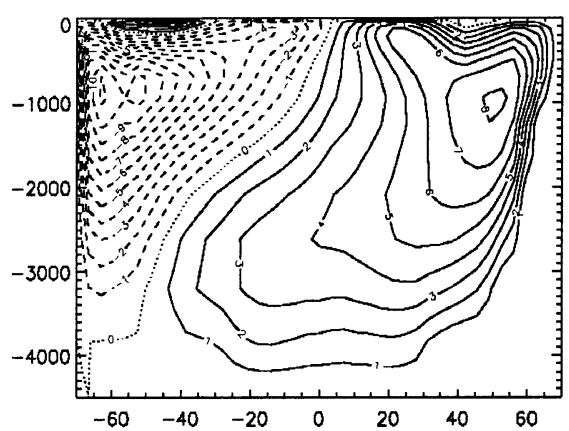

(c)

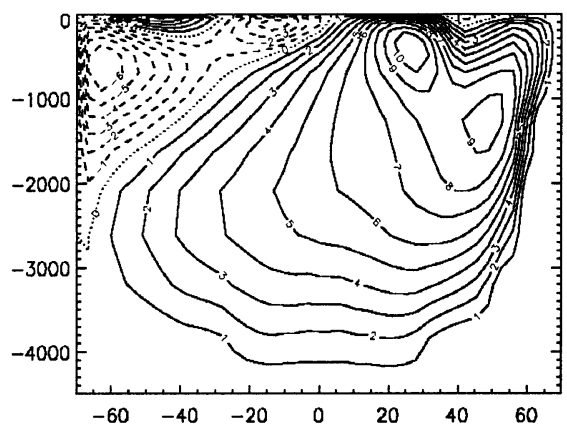

(e)

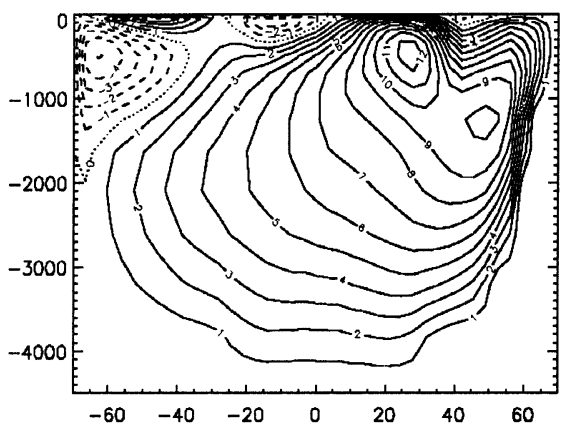

(g)

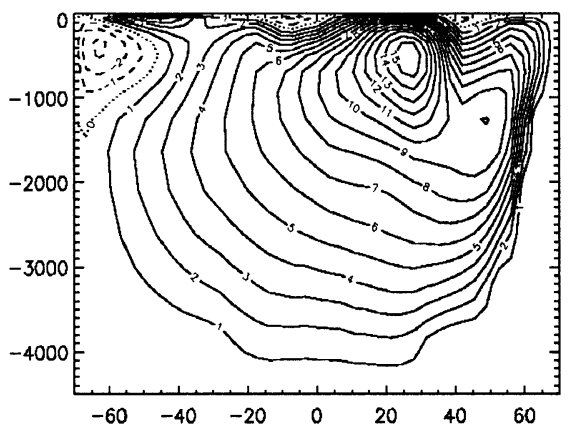

(b)

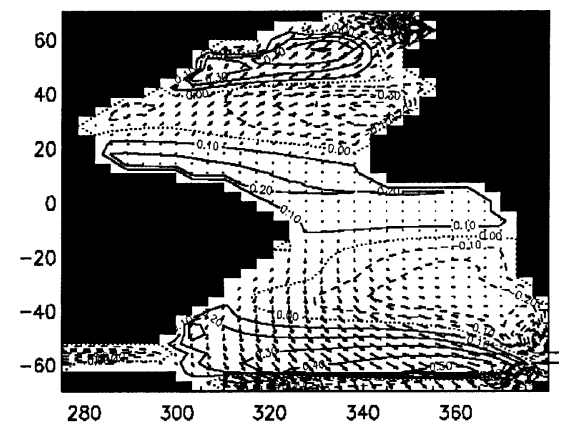

(d)

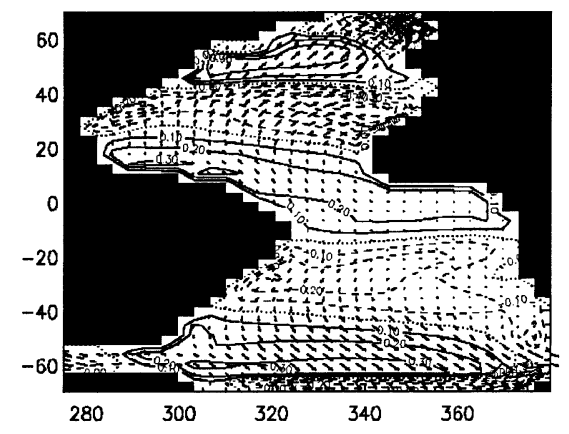

(f)
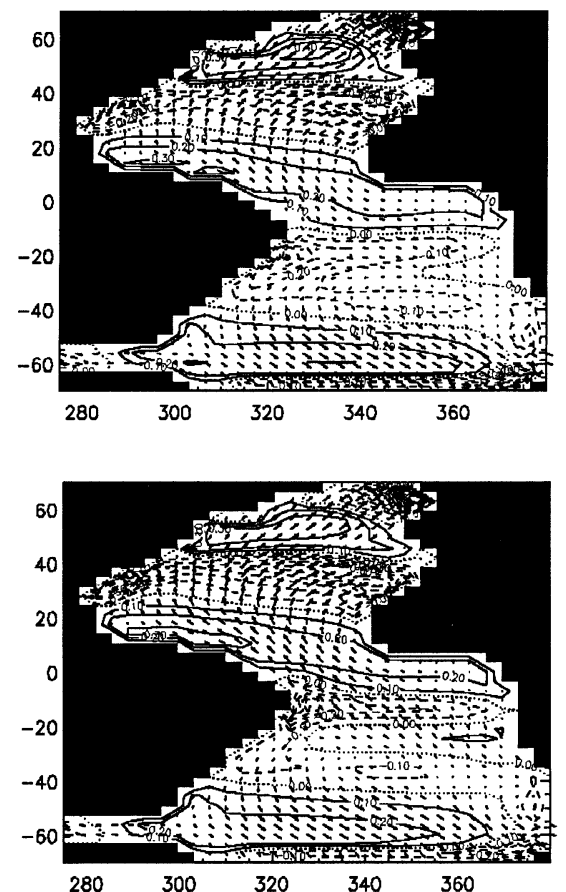

(h)

FIG. 8. Patterns of the meridional overturning streamfunction and surface velocity field for selected location in Fig. 7. Conventions are as in Fig. 2.

circulation in the Southern Ocean, is dramatic. The southern sinking solution is completely eliminated for values of wind stress forcing even smaller than observed. With increasing wind stress, the branch shrinks through an isola into a single point and finally disappears completely (Figs. 9c,d). The realistic Atlantic configuration case appears consistent with this. Thus it seems clear that the open Southern Ocean with wind forcing is an important factor in the structure of THC equilibria. 

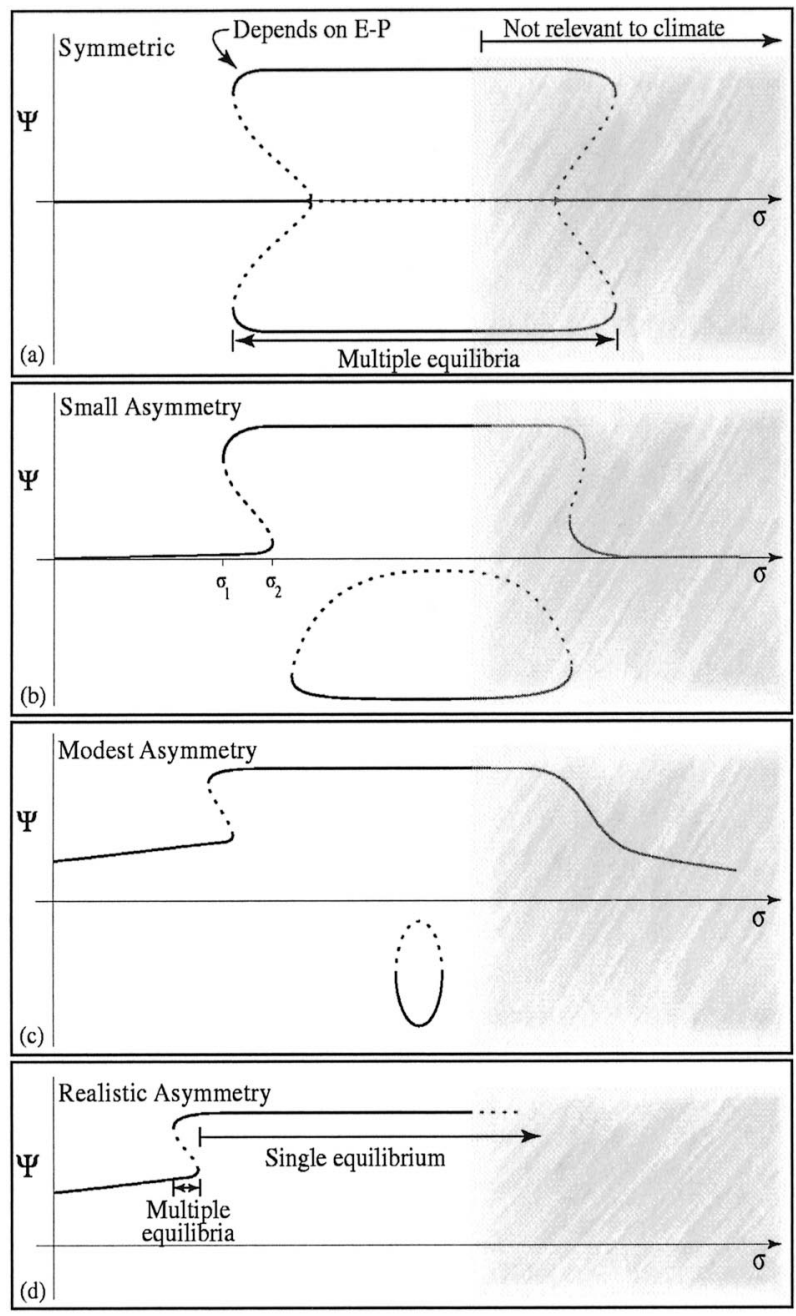

FIG. 9. Sketch of different bifurcation diagrams relating the symmetric closed case to the strongly asymmetric, realistic Atlantic basin case. For each, a measure of the equatorially asymmetric component of the overturning is shown as a function of the freshwater-flux parameter $\sigma$. (a) For latitudinally symmetric boundary conditions. The subcritical pitchfork requires fairly realistic latitude structure of the freshwater flux $E-P$. (b) With a slight latitudinal asymmetry introduced, such as opening the Southern Ocean. The hysteresis bifurcation appears in the interval from $\sigma_{1}$ to $\sigma_{2}$. (c) With larger latitudinal asymmetry, such as including winds (but at smaller than observed strength) on the Southern Ocean. (d) Including all sources of asymmetry. The regions of multiple equilibria and single equilibrium, termed the hysteresis regime and the unique-state regime, respectively, are indicated.

If we consider this asymmetry in context with the ones studied in the two-dimensional models, that is, asymmetric freshwater flux forcing and asymmetric airsea interaction, all these effects tend to reinforce one another to give preference for the northern sinking solution. For the modest asymmetry of opening the Southern Ocean, the qualitative features of the bifurcation diagram are akin to what would occur in the closed basin case with some other slight asymmetry to cause im- perfection. In this respect, this case is similar to those considered in the two-dimensional models although with a new physical mechanism creating the imperfection, namely the presence of the southern zonal flow. In the three-dimensional open case, the effect of the wind stress is a stronger imperfection than those considered in the two-dimensional models, where this branch moved to larger values of the freshwater flux forcing, but remained existent (Dijkstra and Neelin 2000).

In the most asymmetric open configuration considered, one ends up with a single northern sinking branch (Fig. 9d). On this branch, however, multiple equilibria can occur through saddle-node bifurcations, depending on the nature of the pitchfork bifurcation in the equatorially symmetric case. If this pitchfork is a subcritical bifurcation (closed situation) hysteresis occurs on the single branch in the open case. This is similar to the two-dimensional configurations (Dijkstra and Neelin 2000), with another asymmetry causing the imperfection. The sub/supercriticality of the pitchfork bifurcation is shown to depend on the spatial structure of the freshwater flux.

In an equatorially symmetric configuration, the subcritical case is produced not by stronger salt advection feedback but rather by weakening it in a manner that strongly stabilizes the symmetric thermal branch against small amplitude perturbations, while only slightly weakening the feedback when large perturbations are involved. As a result, the pitchfork bifurcation shifts to much larger $\sigma$ values, while the northern and southern sinking branches are only slightly shifted, as summarized in Fig. 10. The difference between subcritical and supercritical pitchfork bifurcations may appear secondary when spontaneous symmetry breaking is considered for a case with equatorially symmetric boundary conditions. The results here show that the subcritical bifurcation on the northern sinking branch is the origin of the hysteresis bifurcation that occurs for equatorially asymmetric conditions; this is consistent with the results in Klinger and Marotzke (1999).

In the realistic geometry case, the southern sinking branch has been disfavored by four mechanisms that act in the same direction: (i) the continental asymmetry effect on atmospheric forcing relative to ocean heat transport (Dijkstra and Neelin 2000); (ii) the imperfection due to open southern channel; (iii) the wind stress on the ACC, the impacts of which were seen in the idealized geometry case above; and (iv) the asymmetry in the freshwater flux. Because the asymmetry is so strong in this realistic configuration and because all tendencies are in the same direction, we do not attempt to quantify relative importance of the different effects as was done in the idealized geometry case.

Assessing the overall comparison with two-dimensional models for the features explored here, many of 


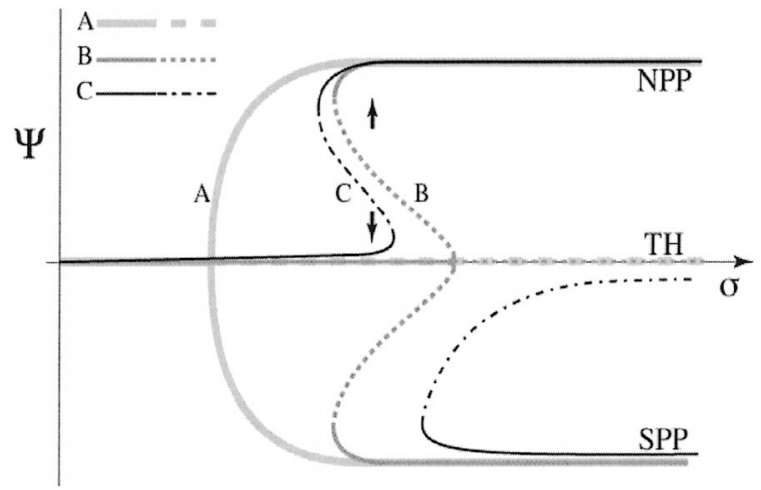

FIG. 10. Schematic summarizing the origin of the hysteresis bifurcation. Three bifurcation diagrams representing the conceptual steps are overlaid. For each, the equatorially asymmetric component of the overturning is shown as a function of the freshwater-flux parameter $\sigma$. Curve A is for a latitudinally symmetric configuration with a simplified freshwater flux for which the salinity advection feedback yields a supercritical pitchfork bifurcation. Curve B is for a symmetric configuration but with a more realistic latitude dependence of the freshwater flux. The symmetric state becomes much more stable, especially to small-amplitude perturbations (arrows denote direction of movement toward equilibrium) resulting in a large shift of the pitchfork bifurcation toward larger $\sigma$. Finite-amplitude NPP and SPP solutions are also shifted but less so, yielding a subcritical pitchfork. In curve $\mathrm{C}$, the latitudinal asymmetries are included that favor the NPP branch. The northern-sinking side of the subcritical pitchfork yields the hysteresis bifurcation that persists in realistic configurations.

the qualitative properties of the bifurcation diagrams are found in the two-dimensional models. As far as this qualitative behavior is concerned, the two-dimensional models indeed provide much guidance. The impact of the ACC and quantitative assessment of the hysteresis regimes, where the three-dimensionality of the flow is important, appear to be in areas where the two-dimensional models would not be likely to give the correct sensitivity to parameters. The large impact of the wind in the open Southern Ocean on the bifurcation diagram in the results suggests that the presence of multiple equilibria in models without proper representations of these processes must be viewed with caution, especially with respect to southern sinking solutions.

To summarize the complete connection between the equatorially symmetric case and the realistic Atlantic case, drawing on results from other bifurcation studies (Weijer et al. 1999; Dijkstra and Neelin 2000) as well as the present results, we refer again to Fig. 9. With sufficient structure in the freshwater flux, the pitchfork bifurcation in the symmetric case is subcritical. The opening of the southern channel, combined with winds, creates the isola that disappears under strong enough wind forcing. What remains in the realistic parameter range (Fig. 9d) is a fairly large regime where the northern sinking branch is unique, termed the unique-state regime, together with a smaller regime of multiple equilibria, termed the hysteresis regime. Overall, the conceptual picture shifts from a bifurcation diagram informed by the idealized, symmetric case in which multiple equilibria are rampant in the parameter space to a rather different view. All asymmetry mechanisms favor the northern Atlantic sinking branch to such an extent that a Pole-to-Pole circulation dominated by southern sinking does not exist in most of the relevant part of parameter space.

Multiple equilibria are entirely associated with the hysteresis bifurcation that has either weaker or stronger northern sinking. If this hysteresis regime occurs in the realistic part of parameter space it can have strong impacts on paleoclimate and global warming (Broecker 1995). However, it exists in a very particular parameter interval that is more restricted than the multiple equilibria suggested in idealized geometry or symmetric studies. It turns out that the hysteresis on the NPP/TH branch is sensitive to a variety of factors, including the spatial structure of $F_{S}$, the geometry, the diffusivity parameters, and the wind stress field. The question of its relevance thus becomes much more quantitative.

Acknowledgments. The authors thank Joyce Meyerson for producing some of the figures in this paper. This work was initiated and finished during several visits of HD to UCLA. Support for these visits has been provided by National Science Foundation Grant ATM-0082529 and by the Institute for Geophysics and Planetary Physics (IGPP). Partial support is from the Netherlands Organization for Scientific Research (NWO) under a PIONIER grant to the first author. Use of the computing facilities was sponsored by the National Computing Facilities Foundation (NCF) with financial support from NWO.

\section{APPENDIX A}

\section{Ocean Model Equations}

The governing equations are the Navier-Stokes equations on the sphere, under the shallow-water approximation, together with the conservation equations of mass, heat, and salt and the linear equation of state. These are

$$
\begin{aligned}
\frac{D u}{d t}-\frac{u v \tan \theta}{r_{0}}-2 \Omega v \sin \theta= & -\frac{1}{\rho_{0} r_{0} \cos \theta} \frac{\partial p}{\partial \phi}+A_{V} \frac{\partial^{2} u}{\partial z^{2}} \\
& +A_{H}\left(\nabla_{H}^{2} u-\frac{u}{r_{0}^{2} \cos ^{2} \theta}-\frac{2 \sin \theta}{r_{0}^{2} \cos ^{2} \theta} \frac{\partial v}{\partial \phi}\right)+\alpha_{\tau} G(z) \frac{\tau_{0}}{\rho_{0} H_{m}} \tau^{\phi}
\end{aligned}
$$




$$
\begin{aligned}
& \frac{D v}{d t}+\frac{u^{2} \tan \theta}{r_{0}}+2 \Omega u \sin \theta=-\frac{1}{\rho_{0} r_{0}} \frac{\partial p}{\partial \theta}+A_{V} \frac{\partial^{2} v}{\partial z^{2}}+A_{H}\left(\nabla_{H}^{2} v-\frac{v}{r_{0}^{2} \cos ^{2} \theta}+\frac{2 \sin \theta}{r_{0}^{2} \cos ^{2} \theta} \frac{\partial u}{\partial \phi}\right) \\
& +\alpha_{\tau} G(z) \frac{\tau_{0}}{\rho_{0} H_{m}} \tau^{\theta}, \\
& \frac{\partial p}{\partial z}=-\rho g, \\
& \frac{\partial w}{\partial z}+\frac{1}{r_{0} \cos \theta}\left[\frac{\partial u}{\partial \phi}+\frac{\partial(v \cos \theta)}{\partial \theta}\right]=0 \\
& \frac{D T}{d t}=\nabla_{H} \cdot\left(K_{N} \nabla_{H} T\right)+\frac{\partial}{\partial z}\left(K_{V} \frac{\partial T}{\partial z}\right)+G(z) \frac{Q_{o a}}{\rho_{0} C_{p o} \tau_{T}}, \\
& \frac{D S}{d t}=\nabla_{H} \cdot\left(K_{H} \nabla_{H} S\right)+\frac{\partial}{\partial z}\left(K_{V} \frac{\partial S}{\partial z}\right)+G(z) \frac{F_{0}}{H_{m}} F_{S}, \quad \text { and } \\
& \rho=\rho_{0}\left[1-\alpha_{T}\left(T-T_{0}\right)+\alpha_{S}\left(S-S_{0}\right)\right],
\end{aligned}
$$

with

$$
\begin{aligned}
\frac{D F}{d t} & =\frac{\partial F}{\partial t}+\frac{u}{r_{0} \cos \theta} \frac{\partial F}{\partial \phi}+\frac{v}{r_{0}} \frac{\partial F}{\partial \theta}+w \frac{\partial F}{\partial z}, \\
\boldsymbol{\nabla}_{H} F & =\left(\frac{1}{r_{0} \cos \theta} \frac{\partial F}{\partial \phi}, \frac{1}{r_{0}} \frac{\partial F}{\partial \theta}\right), \text { and } \\
\boldsymbol{\nabla}_{H} \cdot \mathbf{F} & =\frac{1}{r_{0} \cos \theta}\left[\frac{\partial}{\partial \phi} F^{\phi}+\frac{\partial}{\partial \theta}\left(F^{\theta} \cos \theta\right)\right],
\end{aligned}
$$

where $F$ and $\mathbf{F}=\left(F^{\phi}, F^{\theta}\right)$ are an arbitrary scalar and vector.

The function $G(z)$ is only 1 in the first level of the ocean and is 0 otherwise and is used to model the surface forcing as a body force. Two types of "convective adjustment" are implemented (Dijkstra et al. 2001). One is a local adjustment, in which the vertical mixing coefficient $K_{V}$ is enhanced in regions where dense water overlies lighter water. The other is a complete mixing scheme, called the Global Adjustment Procedure (Weijer and Dijkstra 2001), where iteratively the stably stratified solution is found from the unstably stratified one.

\section{APPENDIX B}

\section{Physics of the Sub/Supercritical Pitchfork}

A more formal way to explain the existence of the subcritical pitchfork is in terms of classical finite amplitude instabilities. Close to the pitchfork, the symmetric state is still stable to infinitesimally small perturbations, while particular finite-amplitude perturbations exist that are able to extract energy of the symmetric state; it is said to be conditionally stable (Joseph 1976). The energetics of the linear instability was considered in Weijer and Dijkstra (2001), where it was shown that the pitchfork bifurcation can be characterized by the zero-crossing of a particular energy functional; that is,

$$
\frac{d \mathcal{E}}{d t}=\frac{1}{2} \frac{d\left\langle\tilde{\rho}^{2}\right\rangle}{d t}=0
$$

where $\rho$ is density, $(\sim)$ denotes a perturbation from the steady state, in this case the symmetric state, and \langle\rangle indicates integration over the flow domain. In the linear stability problem, the spatial pattern of the relevant perturbation is provided by an eigenvector. It was found that the energy producing term is associated with the salinity advection feedback, represented by the term $\langle\tilde{\rho} \tilde{\mathbf{u}} \cdot \nabla \bar{S}\rangle$, where $\bar{S}$ is the salinity field of the symmetric solution. In a conditionally stable regime, the advection of $\bar{S}$ is destabilizing but not sufficiently to overcome the damping effect. Hence, nonlinear terms are needed to explain the finite-amplitude instability and nonlinear effects in the salinity advection feedback are a potential extra source of energy; in the tendency of the energy functional, these show up as $\langle\tilde{\rho} \tilde{\mathbf{u}} \cdot \nabla \tilde{S}\rangle$.

Consider the term $\langle-\tilde{\rho} \tilde{\mathbf{u}} \cdot \nabla(\bar{S}+\tilde{S})\rangle$, which will favor growth of perturbation energy when positive. Assume now, as in section 5 , a perturbation $\tilde{S}$ and $\tilde{\rho}$ that are positive in the Northern Hemisphere with $\tilde{v}$ northward at the surface, as would be typical of the eigenmode associated with the bifurcation. In the case of the flux $F_{S}^{1}, \bar{S}$ is maximum in the Tropics with a single sign of the gradient over most of the hemisphere. While the integral also depends on the salinity structure at depth, it is clear that northward anomalous advection of surface salinity will correlate with $\tilde{\rho}$ to increase density in the north, favoring growth. When $\bar{S}$ has a tropical minimum, on the other hand, and the gradient of $\bar{S}$ changes sign in the subtropics, the situation is much less favorable. 
Consider a solution in the conditionally stable region. In that case, the linear term $\langle-\tilde{\rho} \tilde{\mathbf{u}} \cdot \nabla \bar{S}\rangle$ is too small to overcome damping effects. However, the overturning anomaly $\tilde{\mathbf{u}}$ will advect salty waters from the subtropical Southern Hemisphere northward, increasing the salinity near the equator, so that $\tilde{S}>0$ there and the gradient in each hemisphere correlates better with the large-scale antisymmetric density anomaly. If the $\tilde{S}$ contribution to salinity gradient is sufficiently large, $\langle-\tilde{\rho} \tilde{\mathbf{u}} \cdot \nabla \tilde{S}\rangle$ may just provide the amount of energy required to overcome the threshold implied by the damping terms. This would make the instability grow and would lead to a selfmaintaining equilibrium in the finite-amplitude pole-topole solution.

\section{REFERENCES}

Bice, K., and J. Marotzke, 2001: Numerical evidence against reversed thermohaline circulation in the warm Paleocene/Eocene ocean. J. Geophys. Res., 106, 11 529-11 542.

Broecker, W. S., 1995: The Glacial World According to Wally. Eldigio Press, $318 \mathrm{pp}$.

Bryan, F. O., 1986: High-latitude salinity effects and interhemispheric thermohaline circulations. Nature, 323, 301-304.

Cessi, P., and W. R. Young, 1992: Multiple equilibria in two-dimensional thermohaline circulation. J. Fluid Mech., 241, 291-309.

Dijkstra, H. A., 2000: Nonlinear Physical Oceanography: A Dynamical Systems Approach to the Large Scale Ocean Circulation and El Niño. Kluwer Academic, $456 \mathrm{pp}$.

_ , and M. J. Molemaker, 1997: Symmetry breaking and overturning oscillations in thermohaline-driven flows. J. Fluid Mech., 331, 195-232.

— culation: Latitudinal asymmetry versus asymmetric freshwater flux. J. Climate, 13, 366-382.

— , H. Öksüzŏglu, F. W. Wubs, and E. F. F. Botta, 2001: A fully implicit model of the three-dimensional thermohaline ocean circulation. J. Comput. Phys., 176, 685-715.

Fanning, A. F., and A. J. Weaver, 1996: An atmospheric energymoisture balance model: Climatology, interpentadecal change and coupling to an ocean general circulation model. J. Geophys. Res., 101, 15 111-15 128.

Ganopolsky, A., S. Rahmstorf, V. Petoukhov, and M. Claussen, 2001: Rapid changes of glacial climate simulated in a coupled climate model. Nature, 409, 153-158.

Joseph, D. D., 1976: Stability of Fluid Motions. Vol. 1, SpringerVerlag, $282 \mathrm{pp}$.

Klinger, B. A., and J. Marotzke, 1999: Behavior of double-hemispheric thermohaline flows in a single basin. J. Phys. Oceanogr., 29, 382-399.

Levitus, S., R. Burgett, and T. Boyer, 1994: Salinity. Vol. 3, World Ocean Atlas 1994, NOAA Atlas NESDIS 3, 99 pp.

Manabe, S., and R. J. Stouffer, 1988: Two stable equilibria of a coupled ocean-atmosphere model. J. Climate, 1, 841-866.

Marotzke, J., 2000: Abrupt climate change and thermohaline circu- lation: Mechanisms and predictability. Proc. Natl. Acad. Sci., 97, 1347-1350.

, and P. Willebrand, 1991: Multiple equilibria of the global thermohaline circulation. J. Phys. Oceanogr., 21, 1372-1385.

__ P. Welander, and J. Willebrand, 1988: Instability and multiple steady states in a meridional-plane model of thermohaline circulation. Tellus, 40, 162-172.

McAvaney, B., 2001: Model evaluation. Climate Change 2001: The Scientific Basis, J. T. Houghton et al., Eds., Cambridge University Press, 225-256.

Nayfeh, A. H., and B. Balachandran, 1995: Applied Nonlinear Dynamics. John Wiley and Sons, $685 \mathrm{pp}$.

North, G. R., R. F. Cahalan, and J. A. Coakley, 1981: Energy balance climate models. Rev. Geophys. Space Phys., 19, 19-121.

Quon, C., and M. Ghil, 1992: Multiple equilibria in thermosolutal convection due to salt-flux boundary conditions. J. Fluid Mech., $\mathbf{2 4 5}, 449-484$

Rahmstorf, S., 1995: Multiple convection patterns and thermohaline flow in an idealized OGCM. J. Climate, 8, 3028-3039.

_- 1996: On the freshwater forcing and transport of the Atlantic thermohaline circulation. Climatic Dyn., 12, 799-811.

_ 2000: The thermohaline circulation: A system with dangerous thresholds. Climatic Change, 46, 247-256.

—_, and M. England, 1997: Influence of Southern Hemisphere winds on North Atlantic Deep Water flow. J. Phys. Oceanogr., 27, 2040-2054.

Seydel, R., 1994: Practical Bifurcation and Stability Analysis: From Equilibrium to Chaos. Springer-Verlag, $407 \mathrm{pp}$.

Spall, M., and R. S. Pickart, 2001: Where does dense water sink? A subpolar gyre example. J. Phys. Oceanogr., 31, 810-826.

Stocker, T. F., 2000: Past and future reorganisations in the climate system. Ouat. Sci. Rev., 19, 301-319.

Stommel, H., 1961: Thermohaline convection with two stable regimes of flow. Tellus, 13, 244-230.

Thual, O., and J. C. McWilliams, 1992: The catastrophe structure of thermohaline convection in a two-dimensional fluid model and a comparison with low-order box models. Geophys. Astrophys. Fluid Dyn., 64, 67-95.

Toggweiler, J. R., and B. Samuels, 1995: Effect of Drake Passage on the global thermohaline circulation. Deep-Sea Res., 42, 477500 .

Trenberth, K. E., J. G. Olson, and W. G. Large, 1989: A global ocean wind stress climatology based on ECMWF analyses. National Center for Atmospheric Research Tech. Rep. NCAR/TN$338+$ STR, Boulder, CO, 93 pp

Tziperman, E., 2000: Proximity of the present-day thermohaline circulation to an instability threshold. J. Phys. Oceanogr., 30, 90104.

Vellinga, M., 1998: Multiple equilibria of the thermohaline circulation as a side effect of convective adjustment. J. Phys. Oceanogr., 28, 305-319.

Weijer, W., and H. A. Dijkstra, 2001: Bifurcations of the three-dimensional thermohaline circulation: The double hemispheric case. J. Mar. Res., 59, 599-631.

—_ W. P. M. De Ruijter, H. A. Dijkstra, and P. J. Van Leeuwen, 1999: Impact of interbasin exchange on the Atlantic overturning circulation. J. Phys. Oceanogr., 29, 2266-2284.

,$- \ldots$, and -2001 : Stability of the Atlantic overturning circulation: Competition between Bering Strait freshwater flux and Agulhas heat and salt sources. J. Phys. Oceanogr., 31, 23852402 . 